DOI 10.4171/JEMS/341

Christoph Haberl

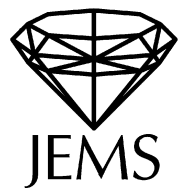

\title{
Minkowski valuations intertwining with the special linear group
}

Received April 25, 2010 and in revised form June 7, 2011

Abstract. All continuous Minkowski valuations which are compatible with the special linear group are completely classified. One consequence of these classifications is a new characterization of the projection body operator.

\section{Introduction}

Ever since they played a critical role in Dehn's solution of Hilbert's Third Problem, valuations have been a central focus in convex geometric analysis (see, e.g., [34, 50,51]). In addition to the ongoing research concerning scalar-valued valuations on convex sets (see, e.g., $[1-4,8,11,15,20,33,36,40,41])$, the study of valuations headed mainly in two different directions during the last years. First, a theory of valuations which are defined on much more general objects than convex sets emerged (see, e.g., [5-7, 9, 10, 12-14,31,32, 63]). Second, valuations with values other than scalars have been characterized (see, e.g., [24-26, 29, 30, 35, 37-39, 56-58]). In particular, Ludwig [35, 37-39] developed a theory of body-valued valuations which are compatible with the whole general linear group (see also [24-26, 58]). She thereby obtained simple characterizations of basic geometric operators. Recently, her results led to strengthenings and generalizations of several affine isoperimetric and Sobolev inequalities [27,28,47,48]. All proofs of such characterizations heavily rely on the assumption of homogeneity.

A central question in the subject has long been: Are the homogeneity assumptions necessitated only by the techniques used in the proofs? This is the first paper to indicate that this indeed may be the case.

Let $\mathcal{K}^{n}$ denote the set of convex bodies in $\mathbb{R}^{n}$ (i.e., non-empty compact convex subsets of $\mathbb{R}^{n}$ ) and write $\mathcal{K}_{o}^{n}$ for the convex bodies containing the origin. We will view $\mathcal{K}^{n}$ as equipped with the Hausdorff metric. The Minkowski sum $K+L$ of two convex bodies $K, L \in \mathcal{K}^{n}$ is the usual vector sum of $K$ and $L$. A Minkowski valuation is a map Z : $\mathcal{K}_{o}^{n} \rightarrow\left\langle\mathcal{K}_{o}^{n},+\right\rangle$ such that

$$
\mathrm{Z}(K \cup L)+\mathrm{Z}(K \cap L)=\mathrm{Z} K+\mathrm{Z} L
$$

C. Haberl: Vienna University of Technology, Wiedner Hauptstraße 8-10, 1040 Vienna, Austria; e-mail: christoph.haberl@tuwien.ac.at 
whenever the union of $K, L \in \mathcal{K}_{o}^{n}$ is again convex. A map Z $: \mathcal{K}_{o}^{n} \rightarrow \mathcal{K}_{o}^{n}$ is said to be $\operatorname{SL}(n)$ contravariant if

$$
\mathrm{Z}(\phi K)=\phi^{-t} \mathrm{Z} K \quad \text { for every } \phi \in \mathrm{SL}(n) \text { and } K \in \mathcal{K}_{o}^{n} \text {. }
$$

Here, $\phi^{-t}$ denotes the inverse of the transpose of $\phi$. One of the main results in this paper shows that for $n \geq 3$ there exists (up to scalar multiples) a unique continuous $\operatorname{SL}(n)$ contravariant Minkowski valuation.

Theorem 1. A map Z : $\mathcal{K}_{o}^{n} \rightarrow\left\langle\mathcal{K}_{o}^{n},+\right\rangle$ is a continuous $\mathrm{SL}(n)$ contravariant Minkowski valuation if and only if there exists a non-negative constant $c$ such that

$$
\mathrm{Z} K=c \Pi K \quad \text { for every } K \in \mathcal{K}_{o}^{n} \text {. }
$$

The projection body $\Pi K$ of $K$ is defined via its support function (see Section 2 for details) by

$$
h_{\Pi K}(u)=\operatorname{vol}_{n-1}\left(K \mid u^{\perp}\right), \quad u \in S^{n-1},
$$

where $\operatorname{vol}_{n-1}$ denotes $(n-1)$-dimensional volume and $K \mid u^{\perp}$ denotes the image of the orthogonal projection of $K$ onto the subspace orthogonal to $u$.

Under the additional assumption of homogeneity in Theorem 1, a characterization of the projection body operator was previously given by Ludwig [38]. Projection bodies were introduced by Minkowski at the turn of the previous century and have since become a central notion in convex geometry. They also arise naturally in a number of different areas such as Minkowski geometry, geometric tomography, symbolic dynamics, and Sobolev inequalities (see, e.g., [17, 21, 27, 45, 61, 62, 66]).

A map Z : $\mathcal{K}_{o}^{n} \rightarrow \mathcal{K}_{o}^{n}$ is said to be $\operatorname{SL}(n)$ covariant if

$$
\mathrm{Z}(\phi K)=\phi \mathrm{Z} K \quad \text { for every } \phi \in \mathrm{SL}(n) \text { and } K \in \mathcal{K}_{o}^{n} .
$$

We also establish the following characterization for $n \geq 3$.

Theorem 2. A map $\mathrm{Z}: \mathcal{K}_{o}^{n} \rightarrow\left\langle\mathcal{K}_{o}^{n},+\right\rangle$ is a continuous $\mathrm{SL}(n)$ covariant Minkowski valuation if and only if there exist non-negative constants $c_{1}, \ldots, c_{4}$ such that

$$
\mathrm{Z} K=c_{1} K+c_{2}(-K)+c_{3} \Gamma_{+} K+c_{4} \Gamma_{+}(-K) \quad \text { for every } K \in \mathcal{K}_{o}^{n} .
$$

The asymmetric centroid body $\Gamma_{+} K$ of $K$ is the convex body whose support function is given by

$$
h_{\Gamma_{+} K}(u)=\int_{K}(u \cdot x)_{+} d x, \quad u \in S^{n-1} .
$$

Here, integration is with respect to Lebesgue measure and $(u \cdot x)_{+}$denotes the positive part of the standard Euclidean product of $u$ and $x$. Note that the definition of the centroid body given above differs from the classical one by a dilation since it is not volume-normalized.

The identity and the centroid body operator were characterized by Ludwig [38] as SL $(n)$ covariant Minkowski valuations which are positively homogeneous. For symmetric bodies, centroid bodies are a classical concept which goes back to Dupin. A decade ago, a 
study of their symmetric analogs was begun (see, e.g., $[16,42,49]$ ) within the $L_{p}$ BrunnMinkowski theory (see, e.g., [16, 17, 19,23, 24, 26-28, 37, 38,42-45,49, 54, 59, 60, 64, 65]). They became objects of interest in asymptotic geometric analysis (see, e.g., [18, 19,53]), information theory (see, e.g., [46]), and even the theory of stable distributions (see, e.g., [52]).

In fact, we will prove more general characterizations of Minkowski valuations than those of Theorems 1 and 2. These results (see Section 3) deal with Minkowski valuations which are either defined on polytopes and are not necessarily continuous on the whole domain, or their images do not have to contain the origin.

\section{Notation and preliminaries}

For quick later reference we develop some notation and basic facts about convex bodies. General references for the theory of convex bodies are provided by the books of Gardner [21], Gruber [22], Schneider [55], and Thompson [62].

We write $\mathbb{R}_{+}$for the set of positive real numbers. The positive and negative parts of a real number $a$ are defined by

$$
(a)_{+}=\max \{a, 0\} \quad \text { and } \quad(a)_{-}=\max \{-a, 0\} .
$$

Critical for us will be the solution to Cauchy's functional equation

$$
f(x+y)=f(x)+f(y) .
$$

Let $f: \mathbb{R} \rightarrow \mathbb{R}$ be a function which satisfies (1) for all $x, y \in \mathbb{R}$ but is not linear. It is well known that the graph of such a function is a dense subset of $\mathbb{R}^{2}$. Every $f: \mathbb{R}_{+} \rightarrow \mathbb{R}$ which satisfies (1) only for positive real numbers can be extended (as an odd function) to a function which satisfies (1) for all $x, y \in \mathbb{R}$. We therefore obtain the following. If $f: \mathbb{R}_{+} \rightarrow \mathbb{R}$ is bounded from below on some non-empty open interval $I \subset \mathbb{R}_{+}$, then

$$
f(x+y)=f(x)+f(y) \forall x, y \in \mathbb{R}_{+} \Rightarrow f(x)=x f(1) \forall x \in \mathbb{R}_{+} .
$$

The letter $n$ will always denote an integer greater than one. Our setting will be Euclidean $n$-space $\mathbb{R}^{n}$. We write $V$ for Lebesgue measure on $\mathbb{R}^{n}$. The standard basis vectors of $\mathbb{R}^{n}$ are denoted by $e_{1}, \ldots, e_{n}$. We write $x_{1}, \ldots, x_{n}$ for the coordinates of a vector $x \in \mathbb{R}^{n}$ with respect to the standard basis. The standard Euclidean inner product of two vectors $x, y \in \mathbb{R}^{n}$ is denoted by $x \cdot y$. Write $\|\cdot\|$ for the norm induced by this inner product and let $S^{n-1}$ denote the Euclidean unit sphere, i.e. the set $\left\{x \in \mathbb{R}^{n}:\|x\|=1\right\}$. For a unit vector $u \in S^{n-1}$, we write $u^{\perp}$ for its orthogonal complement. The linear and convex hull are denoted by lin and conv, respectively. As usual, we write GL( $n)$ for the general linear group and $\operatorname{SL}(n)$ for the special linear group.

A convex body is a non-empty compact convex subset of $\mathbb{R}^{n}$. We write $\mathcal{K}^{n}$ for the set of convex bodies in $\mathbb{R}^{n}$ and denote by $\mathcal{K}_{o}^{n}$ the subset of convex bodies containing the origin. The dimension of a convex body is denoted by dim. Convex polytopes in $\mathbb{R}^{n}$ are denoted by $\mathcal{P}^{n}$ and we write $\mathcal{P}_{o}^{n}$ for the convex polytopes containing the origin. Two 
special polytopes will be used frequently. First, a line segment is the convex hull of two distinct points in $\mathbb{R}^{n}$. The line segment joining distinct points $x, y \in \mathbb{R}^{n}$ is denoted by

$$
[x, y]=\operatorname{conv}\{x, y\} .
$$

Second, the $n$-dimensional standard simplex $T^{n} \in \mathcal{P}_{o}^{n}$ is given by

$$
T^{n}=\operatorname{conv}\left\{o, e_{1}, \ldots, e_{n}\right\} .
$$

A convex body $K \subset \mathcal{K}^{n}$ is uniquely determined by its support function $h_{K}: \mathbb{R}^{n} \rightarrow \mathbb{R}$, where $h_{K}(x)=\max \{x \cdot y: y \in K\}$ for each $x \in \mathbb{R}^{n}$. Note that support functions are sublinear, i.e. for all $x, y \in \mathbb{R}^{n}$ and $\lambda \geq 0$ we have

$$
h_{K}(\lambda x)=\lambda h_{K}(x) \text { and } h_{K}(x+y) \leq h_{K}(x)+h_{K}(y) .
$$

In other words, support functions are positively homogeneous of degree one and subadditive. Conversely, every sublinear function is the support function of a unique convex body. The sublinearity of support functions implies that they are continuous and uniquely determined by their values on the unit sphere $S^{n-1}$.

Next, let us collect three basic properties of support functions. For $K, L \in \mathcal{K}^{n}$ and non-negative numbers $a$ and $b$ we have

$$
h_{a K+b L}=a h_{K}+b h_{L} .
$$

Moreover, if $K \in \mathcal{K}^{n}$ and $\phi \in \mathrm{GL}(n)$ then

$$
h_{\phi K}=h_{K} \circ \phi^{t} .
$$

Suppose that $K \in \mathcal{K}^{n}$ is contained in $\operatorname{lin}\left\{e_{1}, \ldots, e_{k}\right\}$. Then

$$
h_{K}(x)=h_{K}\left(x_{1} e_{1}+\cdots+x_{k} e_{k}\right), \quad x \in \mathbb{R}^{n} .
$$

We need the precise form of support functions of line segments and the standard simplex. The support function of the line segment $[o, v]$ joining the origin and a non-zero point $v \in \mathbb{R}^{n}$ is given by

$$
h_{[o, v]}(x)=(x \cdot v)_{+}, \quad x \in \mathbb{R}^{n} .
$$

For the support function of the $n$-dimensional simplex $T^{n}$ we have

$$
h_{T^{n}}(x)=\max \left\{\left(x_{1}\right)_{+}, \ldots,\left(x_{n}\right)_{+}\right\}, \quad x \in \mathbb{R}^{n} .
$$

The set $\mathcal{K}^{n}$ will be viewed as equipped with the Hausdorff metric. The latter can be defined for $K, L \in \mathcal{K}^{n}$ by

$$
\delta(K, L)=\sup _{u \in S^{n-1}}\left|h_{K}(u)-h_{L}(u)\right| .
$$

Note that the set $\mathcal{P}_{o}^{n}$ of polytopes containing the origin is a dense subset of $\mathcal{K}_{o}^{n}$. 
Associated with each convex body $K \in \mathcal{K}^{n}$ is a Borel measure, $S_{K}$, on $S^{n-1}$ called the surface area measure of $K$. It is defined as the unique finite Borel measure on $S^{n-1}$ such that for all $L \in \mathcal{K}^{n}$,

$$
\int_{S^{n-1}} h_{L} d S_{K}=\lim _{\varepsilon \rightarrow 0^{+}} \frac{V(K+\varepsilon L)-V(K)}{\varepsilon} .
$$

The surface area measure of a polytope is a discrete measure which is concentrated on the outer unit normals of its facets. Moreover, if $u \in S^{n-1}$ is an outer unit normal of a facet of $P \in \mathcal{P}^{n}$, then $S_{P}(\{u\})$ equals the $(n-1)$-dimensional volume of this facet.

Surface area measures are weakly continuous with respect to the Hausdorff metric, i.e., if $\left(K_{i}\right)_{i \in \mathbb{N}}$ is a sequence of bodies in $\mathcal{K}^{n}$ then

$$
\lim _{i \rightarrow \infty} K_{i}=K \Rightarrow \lim _{i \rightarrow \infty} S_{K_{i}}=S_{K}, \text { weakly. }
$$

For convex bodies $K, L \in \mathcal{K}^{n}$ with convex union $K \cup L$ we have

$$
S_{K \cup L}+S_{K \cap L}=S_{K}+S_{L} .
$$

Moreover, if $\lambda \geq 0$ and $K \in \mathcal{K}^{n}$, then

$$
S_{\lambda K}=\lambda^{n-1} S_{K} .
$$

Support functions of projection bodies can be expressed by surface area measures. Indeed, for $K \in \mathcal{K}^{n}$ one has

$$
h_{\Pi K}(u)=\int_{S^{n-1}}(u \cdot v)_{+} d S_{K}(v), \quad u \in S^{n-1} .
$$

This representation together with (9)-(12) shows that $\Pi: \mathcal{K}_{o}^{n} \rightarrow \mathcal{K}_{o}^{n}$ is a continuous $\mathrm{SL}(n)$ contravariant Minkowski valuation which is positively homogeneous of degree $n-1$.

Let $\mathcal{Q}^{n}$ be a subset of $\mathcal{K}^{n}$. A map Z : $\mathcal{Q}^{n} \rightarrow\langle\mathcal{G},+\rangle$ with values in an abelian semigroup is called a valuation if

$$
\mathrm{Z}(P \cup Q)+\mathrm{Z}(P \cap Q)=\mathrm{Z} P+\mathrm{Z} Q \quad \text { whenever } P, Q, P \cup Q, P \cap Q \in \mathcal{Q}^{n} .
$$

If the semigroup $\langle\mathcal{G},+\rangle$ is a subsemigroup of $\left\langle\mathcal{K}^{n},+\right\rangle$, then $\mathrm{Z}$ is called a Minkowski valuation. A map Z : $\mathcal{Q}^{n} \rightarrow \mathcal{K}^{n}$ is said to be $\mathrm{SL}(n)$ co- or contravariant if either $\mathrm{Z}(\phi Q)=\phi \mathrm{Z} Q$ for every $\phi \in \mathrm{SL}(n)$ and $Q \in \mathcal{Q}^{n}$, or $\mathrm{Z}(\phi Q)=\phi^{-t} \mathrm{Z} Q$ for every $\phi \in \operatorname{SL}(n)$ and $Q \in \mathcal{Q}^{n}$.

In the remaining part of this section we will construct several Minkowski valuations which are different from the projection body operator and the asymmetric centroid body operator. Suppose that $P \in \mathcal{P}_{o}^{n}$. We define $\mathcal{N}_{o}(P)$ as the set of all outer unit normals of facets of $P$ containing the origin. Equivalently, for a unit vector $u \in S^{n-1}$ we have $u \in \mathcal{N}_{o}(P)$ if and only if $u \cdot x \leq 0$ for all $x \in P$ and $P \cap u^{\perp}$ is $(n-1)$-dimensional. Let $\Pi_{o}: \mathcal{P}_{o}^{n} \rightarrow \mathcal{K}_{o}^{n}$ be defined by

$$
h_{\Pi_{o} P}(u)=\int_{\mathcal{N}_{o}(P)}(u \cdot v)_{+} d S_{P}(v), \quad u \in S^{n-1} .
$$


The operator $\Pi_{o}$ was defined by Ludwig [38]. It follows directly from (12) that $\Pi_{o}$ is positively homogeneous of degree $n-1$. For the reader's convenience we collect other basic properties in the following lemma.

Lemma 1. The map $\Pi_{o}: \mathcal{P}_{o}^{n} \rightarrow \mathcal{K}_{o}^{n}$ is an $\mathrm{SL}(n)$ contravariant valuation.

Proof. For $P \in \mathcal{P}_{o}^{n}$ define a finite Borel measure $\mu_{P}$ on the sphere by

$$
d \mu_{P}=\chi_{\mathcal{N}_{o}(P)} d S_{P}
$$

where $\chi$ denotes the characteristic function. Note that $\mu_{P}$ is a discrete measure with finite support. In fact, it is concentrated on the outer unit normals of facets of $P$ which contain the origin. Clearly we have

$$
h_{\Pi_{o} P}(u)=\int_{S^{n-1}}(u \cdot v)_{+} d \mu_{P}(v), \quad u \in S^{n-1} .
$$

Let $P, Q \in \mathcal{P}_{o}^{n}$ with $P \cup Q \in \mathcal{P}_{o}^{n}$. Note that $\mathcal{N}_{o}(P \cup Q) \cup \mathcal{N}_{o}(P \cap Q)=\mathcal{N}_{o}(P) \cup \mathcal{N}_{o}(Q)$. This together with the valuation property (11) implies that for every $v \in S^{n-1}$,

$$
\mu_{P \cup Q}(\{v\})+\mu_{P \cap Q}(\{v\})=\mu_{P}(\{v\})+\mu_{Q}(\{v\}) .
$$

Since all measures which are involved in the last equation are discrete, we actually have $\mu_{P \cup Q}+\mu_{P \cap Q}=\mu_{P}+\mu_{Q}$. This together with the representation (13) proves that $\Pi_{o}$ is a valuation.

In order to establish the contravariance of $\Pi_{o}$ suppose that $P \in \mathcal{P}_{o}^{n}$ and $\phi \in \operatorname{SL}(n)$. Write $\langle x\rangle=x /\|x\|$ for $x \in \mathbb{R}^{n} \backslash\{o\}$ and denote by $\langle\phi\rangle: S^{n-1} \rightarrow S^{n-1}$ the map with $\langle\phi\rangle(v)=\left\langle\phi^{-t} v\right\rangle$ for each $v \in S^{n-1}$. Clearly, $\langle\phi\rangle$ is a continuous bijection. For every $v \in S^{n-1}$ we have

$$
\left\|\phi^{-t} v\right\| S_{P}(\{v\})=S_{\phi P}(\{\langle\phi\rangle(v)\}) \quad \text { and } \quad v \in \mathcal{N}_{o}(P) \Leftrightarrow\langle\phi\rangle(v) \in \mathcal{N}_{o}(\phi P) .
$$

Write $\langle\phi\rangle \mu_{P}$ for the image measure of $\mu_{P}$ with respect to $\langle\phi\rangle$. Thus

$$
\langle\phi\rangle \mu_{P}(\{v\})=\left\|\phi^{t} v\right\| \mu_{\phi P}(\{v\}) \quad \text { for every } v \in S^{n-1} .
$$

Since all measures involved in the last equality are discrete with finite support we actually have $d \mu_{\phi P}=\left\|\phi^{t} \cdot\right\|^{-1} d\langle\phi\rangle \mu_{P}$. Representation (13) therefore yields $h_{\Pi_{o} \phi P}=$ $h_{\Pi_{o} P} \circ \phi^{-1}$. From (5) we deduce the $\operatorname{SL}(n)$ contravariance of $\Pi_{o}$.

We finish our discussion of the operators $\Pi$ and $\Pi_{o}$ by two simple formulas. Since the volume of the $n$-dimensional standard simplex equals $1 / n$ !, the above interpretation of surface area measures for polytopes yields

$$
h_{\Pi T^{n}}\left(x_{1} e_{1}+x_{2} e_{2}\right)=\frac{1}{(n-1) !}\left[\left(x_{1}+x_{2}\right)_{+}+\left(x_{1}\right)_{-}+\left(x_{2}\right)_{-}\right],
$$

as well as

$$
h_{\Pi_{o} T^{n}}\left(x_{1} e_{1}+x_{2} e_{2}\right)=\frac{1}{(n-1) !}\left[\left(x_{1}\right)_{-}+\left(x_{2}\right)_{-}\right] .
$$


Let $K \in \mathcal{K}^{n}$. The symmetric centroid body $\Gamma K$ of $K$ is defined by

$$
h_{\Gamma K}(u)=\int_{K}|u \cdot x| d x, \quad u \in S^{n-1} .
$$

It is easily seen that $\Gamma K=\Gamma_{+} K+\Gamma_{+}(-K)$. The moment vector $\mathrm{m}(K)$ of $K$ is given by

$$
h_{\mathrm{m}(K)}(u)=\int_{K} u \cdot x d x, \quad u \in S^{n-1} .
$$

Note that the moment vector is indeed an element of $\mathbb{R}^{n}$. Up to normalization, $\mathrm{m}(K)$ is equal to the centroid of $K$. Clearly, both $\Gamma, \mathrm{m}: \mathcal{K}^{n} \rightarrow\left\langle\mathcal{K}^{n},+\right\rangle$ are continuous $\operatorname{SL}(n)$ covariant Minkowski valuations.

Finally, let $P \in \mathcal{P}_{o}^{2}$ and denote by $\mathcal{E}_{o}(P)$ the set of all edges of $P$ containing the origin. We define an operator $\mathrm{E}: \mathcal{P}_{o}^{2} \rightarrow \mathcal{P}_{o}^{2}$ by

$$
\text { E } P= \begin{cases}\{o\}, & \mathcal{E}_{o}(P)=\emptyset, \\ 2 F, & \mathcal{E}_{o}(P)=\{F\}, P=F, \\ F, & \mathcal{E}_{o}(P)=\{F\}, P \neq F, \\ F_{1}+F_{2}, & \mathcal{E}_{o}(P)=\left\{F_{1}, F_{2}\right\} .\end{cases}
$$

Note that $\mathrm{E}: \mathcal{P}_{o}^{2} \rightarrow\left\langle\mathcal{K}_{o}^{2},+\right\rangle$ is an $\operatorname{SL}(2)$ covariant Minkowski valuation.

\section{Classification results for Minkowski valuations}

Throughout this section let $n \geq 3$. In what follows, we state several classifications which are similar to those given in the introduction but hold under weaker assumptions.

\subsection{Contravariant Minkowski valuations}

Let us begin with a result on $\operatorname{SL}(n)$ contravariant Minkowski valuations which are not necessarily continuous.

Theorem 3. If $\mathrm{Z}: \mathcal{P}_{o}^{n} \rightarrow\left\langle\mathcal{K}_{o}^{n},+\right\rangle$ is an $\mathrm{SL}(n)$ contravariant Minkowski valuation, then there exist constants $c_{1}, c_{2}, c_{3}$ with $c_{1} \geq 0$ and $c_{1}+c_{2}+c_{3} \geq 0$ such that

$$
h_{\mathrm{Z} P}=c_{1} h_{\Pi P}+c_{2} h_{\Pi_{o} P}+c_{3} h_{\Pi_{o}(-P)} \quad \text { for every } P \in \mathcal{P}_{o}^{n} .
$$

We remark that there exist constants $c_{1}, c_{2}$, and $c_{3}$ such that $c_{2}$ or $c_{3}$ is negative but $\mathrm{Z}: \mathcal{P}_{o}^{n} \rightarrow\left\langle\mathcal{K}_{o}^{n},+\right\rangle$ defined by (16) is an $\operatorname{SL}(n)$ contravariant Minkowski valuation. Under the assumption of continuity, we have the following characterization of Minkowski valuations whose images do not have to contain the origin a priori.

Theorem 4. A map Z: $\mathcal{K}_{o}^{n} \rightarrow\left\langle\mathcal{K}^{n},+\right\rangle$ is a continuous $\mathrm{SL}(n)$ contravariant Minkowski valuation if and only if there exists a non-negative constant $c$ such that

$$
\mathrm{Z} K=c \Pi K \quad \text { for every } K \in \mathcal{K}_{o}^{n} \text {. }
$$




\subsection{Covariant Minkowski valuations}

For Minkowski valuations defined on polytopes we will prove the following.

Theorem 5. A map Z: $\mathcal{P}_{o}^{n} \rightarrow\left\langle\mathcal{K}_{o}^{n},+\right\rangle$ is an $\mathrm{SL}(n)$ covariant Minkowski valuation which is continuous at the line segment $\left[o, e_{1}\right]$ if and only if there exist non-negative constants $c_{1}, \ldots, c_{4}$ such that

$$
\mathrm{Z} P=c_{1} P+c_{2}(-P)+c_{3} \Gamma_{+} P+c_{4} \Gamma_{+}(-P) \quad \text { for every } P \in \mathcal{P}_{o}^{n} .
$$

Finally, the next result characterizes all continuous $\operatorname{SL}(n)$ covariant Minkowski valuations whose range is $\mathcal{K}^{n}$.

Theorem 6. A map Z: $\mathcal{K}_{o}^{n} \rightarrow\left\langle\mathcal{K}^{n},+\right\rangle$ is a continuous $\mathrm{SL}(n)$ covariant Minkowski valuation if and only if there exist non-negative constants $c_{1}, \ldots, c_{3}$ and a constant $c_{4} \in \mathbb{R}$ such that

$$
\mathrm{Z} K=c_{1} K+c_{2}(-K)+c_{3} \Gamma K+c_{4} \mathrm{~m}(K) \quad \text { for every } K \in \mathcal{K}_{o}^{n} .
$$

\section{Reduction to simplices}

The aim of this section is to show that $\operatorname{SL}(n)$ co- or contravariant Minkowski valuations are actually determined by their values on dilates of the standard simplex.

Let $P \in \mathcal{P}^{n}$ be $n$-dimensional. A finite set $\mathcal{T}_{P}$ of $n$-dimensional simplices is called a triangulation of $P$ if the union of all simplices in $\mathcal{T}_{P}$ equals $P$ and no two simplices intersect in a set of dimension $n$. Suppose that $x \in P$. A starring of $P$ at $x$ is a triangulation such that each simplex in $\mathcal{T}_{P}$ has a vertex at $x$.

If $P \in \mathcal{P}^{n}$ is an $n$-dimensional polytope and $x \in P$, then it is well-known that there exists a starring of $P$ at $x$. Indeed, for $n=1$ this is trivial. Suppose that it is true for $(n-1)$ dimensional polytopes and denote by $F_{j}, j=1, \ldots, k$, the facets of an $n$-dimensional polytope $P$. We choose starrings $\mathcal{T}_{F_{j}}$ of $F_{j}$ for those facets which do not contain the given point $x$. Thus the convex hulls of $x$ and the $(n-1)$-dimensional simplices in $\mathcal{T}_{F_{j}}$ define the desired starring.

A real valued valuation $\mathrm{z}: \mathcal{P}_{o}^{n} \rightarrow\langle\mathbb{R},+\rangle$ is called simple if convex polytopes of dimension less than $n$ are mapped to zero. The following result is a special case of [24, Lemma 3.2]. For the sake of completeness we give its proof here.

Lemma 2. Let $\mathrm{z}: \mathcal{P}_{o}^{n} \rightarrow\langle\mathbb{R},+\rangle$ be a simple valuation. If $\mathrm{z}(S)=0$ for every $n$ dimensional simplex $S$ having one vertex at the origin, then $\mathrm{z}(P)=0$ for every $P \in \mathcal{P}_{o}^{n}$.

Proof. By what we have seen above, every $P \in \mathcal{P}_{o}^{n}$ has a starring at $o$. Therefore, it suffices to prove that for an $n$-dimensional polytope $P \in \mathcal{P}_{o}^{n}$, the condition

$$
P=P_{1} \cup \cdots \cup P_{k}, \quad P_{1}, \ldots, P_{k} \in \mathcal{P}_{o}^{n}, \quad \operatorname{dim}\left(P_{i} \cap P_{j}\right)<n \quad \text { for } i \neq j
$$

implies

$$
\mathrm{z}(P)=\sum_{i=1}^{k} \mathrm{z}\left(P_{i}\right) .
$$


We proceed by induction on $k$. For $k=1$ this is trivial. Assume that $k \geq 2$ and suppose that it is true for at most $k-1$ polytopes. Without loss of generality assume that $\operatorname{dim} P_{1}=n$.

We can suppose that $P_{1}$ has at least one facet $F_{1}$ containing the origin such that $P \cap \operatorname{int}\left(\operatorname{lin} F_{1}\right)^{+} \neq \emptyset$. Here, int $\left(\operatorname{lin} F_{1}\right)^{+}$denotes the interior of the halfspace determined by $\operatorname{lin} F_{1}$ which does not contain $P_{1}$. For if no such facet exists, then $P_{1}=P$, implying $\operatorname{dim} P_{i}<n$ for $i=2, \ldots, k$, and (17) would obviously holds by the simplicity of $\mathrm{z}$.

Let $H_{1}, \ldots, H_{l}$ denote the linear hulls of those facets $F_{1}, \ldots, F_{l}$ of $P_{1}$ which contain the origin and satisfy

$$
P \cap \operatorname{int} H_{i}^{+} \neq \varnothing
$$

for $i=1, \ldots, l$, where int $H_{i}^{+}$denotes the interior of the halfspace determined by $H_{i}$ which does not contain $P_{1}$. For $m=1, \ldots, l$ and $i=1, \ldots, k$ set $P^{0}=P, P_{i}^{0}=P_{i}$, and define

$$
P^{m}=P \cap H_{1}^{-} \cap \cdots \cap H_{m}^{-} \quad \text { and } \quad P_{i}^{m}=P_{i} \cap H_{1}^{-} \cap \cdots \cap H_{m}^{-} .
$$

Note that for each $m=1, \ldots, l$ there exists a point $p^{m}$ such that

$$
p^{m} \in P^{m-1} \cap \operatorname{int} H_{m}^{+} .
$$

Indeed, for $m=1$ this directly follows from (18). For $m>1$ choose a point $x$ in the relative interior of the facet $F_{m}=P_{1} \cap H_{m}$. Then $x \in$ int $H_{i}^{-}$for all $i=1, \ldots, m-1$. By (18) we know that there exists a $p \in P \cap$ int $H_{m}^{+}$. Clearly, the set $[x, p] \backslash\{x\}$ is contained in $P \cap$ int $H_{m}^{+}$. Moreover, points of this set which are sufficiently close to $x$ are contained in int $H_{1}^{-} \cap \cdots \cap$ int $H_{m-1}^{-}$. This proves (19).

Next, we are going to prove that for all $m=1, \ldots, l$,

$$
\mathrm{z}(P)=\mathrm{z}\left(P^{m}\right)+\sum_{i=1}^{k}\left[\mathrm{z}\left(P_{i}\right)-\mathrm{z}\left(P_{i}^{m}\right)\right] .
$$

To this end fix $m$ and set

$$
P^{m,+}=P^{m-1} \cap H_{m}^{+} \quad \text { and } \quad P_{i}^{m,+}=P_{i}^{m-1} \cap H_{m}^{+} .
$$

First, we want to prove

$$
P^{m,+}=P_{2}^{m,+} \cup \cdots \cup P_{k}^{m,+} .
$$

Note that since $P^{m,+}=P_{1}^{m,+} \cup \cdots \cup P_{k}^{m,+}$ it is actually enough to show that $P_{1}^{m,+} \subset$ $P_{2}^{m,+} \cup \cdots \cup P_{k}^{m,+}$. Let $x \in P_{1}^{m,+}=P_{1}^{m-1} \cap H_{m}$. From (19) we infer that the set $\left[x, p^{m}\right] \backslash\{x\}$ has to be contained in $P_{2}^{m,+} \cup \cdots \cup P_{k}^{m,+}$. Thus there exists $i \in\{2, \ldots, k\}$ such that points of $\left[x, p^{m}\right] \backslash\{x\}$ sufficiently close to $x$ are contained in $P_{i}^{m,+}$. The closedness of $P_{i}^{m,+}$ concludes the proof of (21). 
Now (21), the induction assumption, and the simplicity of z prove

$$
\mathrm{z}\left(P^{m,+}\right)=\sum_{i=2}^{k} \mathrm{z}\left(P_{i}^{m,+}\right)=\sum_{i=1}^{k} \mathrm{z}\left(P_{i}^{m,+}\right)=\sum_{i=1}^{k}\left[\mathrm{z}\left(P_{i}^{m-1}\right)-\mathrm{z}\left(P_{i}^{m}\right)\right] .
$$

By the valuation property of $\mathrm{z}$ and its simplicity we therefore obtain

$$
\mathrm{z}\left(P^{m-1}\right)=\mathrm{z}\left(P^{m}\right)+\mathrm{z}\left(P^{m,+}\right)=\mathrm{z}\left(P^{m}\right)+\sum_{i=1}^{k}\left[\mathrm{z}\left(P_{i}^{m-1}\right)-\mathrm{z}\left(P_{i}^{m}\right)\right] .
$$

For $m=1$ this is precisely (20). Let $1<m \leq l$ and assume that (20) holds for $m-1$. Then inserting (22) in this equation immediately shows that (20) also holds for $m$. Inductively we have thus proved (20) for all $m=1, \ldots, l$.

Since $P_{1}^{l}=P^{l}$ we have $\operatorname{dim} P_{i}^{l}<n$ for $i=2, \ldots, k$, and hence

$$
\mathrm{z}\left(P^{l}\right)=\sum_{i=1}^{k} \mathrm{z}\left(P_{i}^{l}\right) .
$$

Inserting this in (20) for $m=l$ proves (17).

Lemma 3. For $i=1, \ldots, k$, let $c_{i} \in \mathbb{R}$ and suppose that $\mathrm{Z}_{i}: \mathcal{P}_{o}^{n} \rightarrow\left\langle\mathcal{K}^{n},+\right\rangle$ are Minkowski valuations. If $\mathrm{Z}_{i}$ 's are either all $\mathrm{SL}(n)$ covariant or all $\mathrm{SL}(n)$ contravariant and

$$
\sum_{i=1}^{k} c_{i} h_{Z_{i}\left(s T^{n}\right)}=0 \quad \text { for every } s>0,
$$

then

$$
\sum_{i=1}^{k} c_{i} h_{\mathrm{Z}_{i} P}=0 \quad \text { for every } P \in \mathcal{P}_{o}^{n} .
$$

Proof. Let $x \in \mathbb{R}^{n}$ and define a map z $: \mathcal{P}_{o}^{n} \rightarrow\langle\mathbb{R},+\rangle$ by

$$
\mathrm{z}(P)=\sum_{i=1}^{k} c_{i} h_{\mathrm{Z}_{i} P}(x) .
$$

From (4) we deduce that $\mathrm{z}$ is a valuation. Let $S$ be an $n$-dimensional simplex with one vertex at the origin. Note that there exists a $\phi \in \operatorname{SL}(n)$ and a positive number $s$ such that $\phi\left(s T^{n}\right)=S$. Since $\mathrm{Z}_{i}$ 's are assumed to be either all $\mathrm{SL}(n)$ covariant or all $\mathrm{SL}(n)$ contravariant, we infer from (5) and (23) that $\mathrm{z}(S)=0$.

Let $0 \leq k<n$ and $S^{\prime}$ be a $k$-dimensional simplex with one vertex at the origin. There exists a $(k+1)$-dimensional simplex $S$ with one vertex at the origin and a hyperplane $H$ such that $S \cap H^{+}$and $S \cap H^{-}$are both $k$-dimensional simplices with one vertex at the origin and $S^{\prime}=S \cap H$. As before, $H^{ \pm}$denote the two halfspaces determined by $H$. The valuation property of $\mathrm{z}$ implies

$$
\mathrm{z}(S)+\mathrm{z}\left(S^{\prime}\right)=\mathrm{z}\left(S \cap H^{+}\right)+\mathrm{z}\left(S \cap H^{-}\right) .
$$

Since $\mathrm{z}$ vanishes on $n$-dimensional simplices with one vertex at the origin, an obvious induction argument shows that $\mathrm{z}$ vanishes on all simplices with one vertex at the origin. 
By induction on the dimension $k$, we will now show that $\mathrm{z}$ vanishes on $k$-dimensional polytopes. For $k=0$ this obviously follows from the results of the last paragraph. Assume that $\mathrm{z}$ vanishes on $k$-dimensional polytopes and let $P \in \mathcal{P}_{o}^{n}$ be $(k+1)$-dimensional. Set $H=\operatorname{lin} P$ and write $\pi: \mathbb{R}^{k+1} \rightarrow H$ for an arbitrary but fixed linear bijection. Then $\hat{\mathrm{z}}: \mathcal{P}_{o}^{k+1} \rightarrow\langle\mathbb{R},+\rangle$ defined by $\hat{\mathrm{z}}(Q)=\mathrm{z}(\pi Q)$ is a simple valuation which vanishes on $(k+1)$-dimensional simplices with one vertex at the origin. Lemma 2 shows that $\hat{\mathrm{z}}=0$. Hence also $\mathrm{z}(P)=0$.

\section{Functional equations}

Throughout this section let $\lambda \in(0,1)$ and $p, q \in \mathbb{R}$. Suppose that functions $g, h$ : $(0,1) \rightarrow \mathbb{R}_{+}$are given. We define two families of linear maps on $\mathbb{R}^{n}$ by

$\phi_{\lambda, g} e_{2}=(1-\lambda) e_{1}+\lambda e_{2}, \quad \phi_{\lambda, g} e_{1}=e_{1}, \quad \phi_{\lambda, g} e_{k}=g(\lambda) e_{k} \quad$ for $3 \leq k \leq n$ if $n \geq 3$,

and

$\psi_{\lambda, h} e_{1}=(1-\lambda) e_{1}+\lambda e_{2}, \quad \psi_{\lambda, h} e_{2}=e_{2}, \quad \psi_{\lambda, h} e_{k}=h(\lambda) e_{k} \quad$ for $3 \leq k \leq n$ if $n \geq 3$.

If $g$ and $h$ are constantly 1 we set $\phi_{\lambda}=\phi_{\lambda, g}$ and $\psi_{\lambda}=\psi_{\lambda, h}$.

The aim of this section is to deduce properties of functions $f: \mathbb{R}_{+} \times \mathbb{R}^{n} \rightarrow \mathbb{R}$ which satisfy at least one of the following three functional equations. The first one is

$$
f(s, x)=\lambda^{p} f\left(s \lambda^{q}, \phi_{\lambda, g}^{t} x\right)+(1-\lambda)^{p} f\left(s(1-\lambda)^{q}, \psi_{\lambda, h}^{t} x\right) .
$$

The second one is just the special case of (24) where $g$ and $h$ are constantly 1 . It can be written as

$$
f(s, x)=\lambda^{p} f\left(s \lambda^{q}, \phi_{\lambda}^{t} x\right)+(1-\lambda)^{p} f\left(s(1-\lambda)^{q}, \psi_{\lambda}^{t} x\right) .
$$

The third one reads

$$
f(s, x)=\lambda^{p} f\left(s \lambda^{q}, \phi_{\lambda}^{-1} x\right)+(1-\lambda)^{p} f\left(s(1-\lambda)^{q}, \psi_{\lambda}^{-1} x\right) .
$$

The statement that $f: \mathbb{R}_{+} \times \mathbb{R}^{n} \rightarrow \mathbb{R}$ satisfies (24) on $A \subset \mathbb{R}^{n}$ has to be understood as follows: There exist $p, q \in \mathbb{R}$ and functions $g, h:(0,1) \rightarrow \mathbb{R}_{+}$such that (24) holds for all $\lambda \in(0,1), s \in \mathbb{R}_{+}$, and $x \in A$. Similarly, $f$ is said to satisfy (25) or (26) on $A \subset \mathbb{R}^{n}$ if there exist $p, q \in \mathbb{R}$ such that (25), respectively (26), holds for all $\lambda \in(0,1), s \in \mathbb{R}_{+}$, and $x \in A$.

\subsection{Homogeneity}

Let us start by showing that a function which solves (25) at certain points is positively homogeneous in its first argument. 
Lemma 4. Let $n \geq 3$. Iffor some $q \neq 0$ the function $f: \mathbb{R}_{+} \times \mathbb{R}^{n} \rightarrow \mathbb{R}$ satisfies equation (25) at the points $\pm e_{3}$ and the functions $f\left(\cdot, e_{3}\right)$ and $f\left(\cdot,-e_{3}\right)$ are bounded from below on some non-empty open intervals $I_{+} \subset \mathbb{R}_{+}$and $I_{-} \subset \mathbb{R}_{+}$, respectively, then

$$
f\left(s, e_{3}\right)=s^{(1-p) / q} f\left(1, e_{3}\right) \quad \text { and } \quad f\left(s,-e_{3}\right)=s^{(1-p) / q} f\left(1,-e_{3}\right)
$$

for every $s>0$.

Proof. Note that $e_{3}$ is a fixpoint of both $\phi_{\lambda}^{t}$ and $\psi_{\lambda}^{t}$. From (25) we see that

$$
f\left(s, e_{3}\right)=\lambda^{p} f\left(s \lambda^{q}, e_{3}\right)+(1-\lambda)^{p} f\left(s(1-\lambda)^{q}, e_{3}\right)
$$

for every $s>0$ and $\lambda \in(0,1)$. Define $g: \mathbb{R}_{+} \rightarrow \mathbb{R}$ by

$$
g(s)=f\left(s^{q}, e_{3}\right) \quad \text { for } s>0 .
$$

Then, for every $s>0$ and $\lambda \in(0,1)$, equation (27) reads

$$
g\left(s^{1 / q}\right)=\lambda^{p} g\left(s^{1 / q} \lambda\right)+(1-\lambda)^{p} g\left(s^{1 / q}(1-\lambda)\right) .
$$

Let $x$ and $y$ be arbitrary positive real numbers. Set

$$
s=(x+y)^{q} \quad \text { and } \quad \lambda=x(x+y)^{-1} .
$$

If we insert these particular values of $s$ and $\lambda$ in (28), then we have, for all $x, y>0$,

$$
(x+y)^{p} g(x+y)=x^{p} g(x)+y^{p} g(y) .
$$

Thus the function $t \mapsto t^{p} g(t)$ solves Cauchy's functional equation (1) on $\mathbb{R}_{+}$and, by assumption, there exists a non-empty open interval $I \subset \mathbb{R}_{+}$where this function is bounded from below. We infer from (2) that $t^{p} g(t)=\operatorname{tg}(1)$ and hence

$$
g(t)=t^{1-p} g(1) \text {. }
$$

Finally, the definition of $g$ immediately yields

$$
f\left(s, e_{3}\right)=g\left(s^{1 / q}\right)=s^{(1-p) / q} g(1)=s^{(1-p) / q} f\left(1, e_{3}\right) .
$$

Replacing $e_{3}$ by $-e_{3}$ in the above derivation concludes the proof of the lemma.

Corollary 1. Let $n \geq 3$. Assume that for some $q \neq 0$ the function $f: \mathbb{R}_{+} \times \mathbb{R}^{n} \rightarrow \mathbb{R}$ satisfies equation (25) at the points $\pm e_{3}$, the functions $f\left(\cdot, e_{3}\right)$ and $f\left(\cdot,-e_{3}\right)$ are bounded from below on some non-empty open intervals $I_{+} \subset \mathbb{R}_{+}$and $I_{-} \subset \mathbb{R}_{+}$, respectively, and

$$
f\left(s, \pi e_{3}\right)=f\left(s, e_{3}\right) \quad \text { and } \quad f\left(s,-\pi e_{3}\right)=f\left(s,-e_{3}\right)
$$

for all $s>0$ and $\pi \in \mathrm{SL}(n)$ induced by a permutation matrix. Then for all $i=1, \ldots, n$ and every $s>0$,

$$
f\left(s, e_{i}\right)=s^{(1-p) / q} f\left(1, e_{i}\right) \quad \text { and } \quad f\left(s,-e_{i}\right)=s^{(1-p) / q} f\left(1,-e_{i}\right) .
$$

Proof. Let $i \in\{1, \ldots, n\}$. Since $n \geq 3$, there exists $\pi \in \operatorname{SL}(n)$ which is induced by a permutation matrix and satisfies $e_{i}=\pi e_{3}$. From our assumption (29) and Lemma 4 we deduce

$$
f\left(s, e_{i}\right)=f\left(s, \pi e_{3}\right)=f\left(s, e_{3}\right)=s^{(1-p) / q} f\left(1, e_{3}\right)=s^{(1-p) / q} f\left(1, e_{i}\right) .
$$

The argument for $-e_{i}$ is similar. 


\subsection{Uniqueness}

In this subsection we deduce that, under certain circumstances, solutions to the equations (25) and (26) are uniquely determined by their values on a 2-dimensional subspace.

Lemma 5. Let $n \geq 3$ and $f: \mathbb{R}_{+} \times \mathbb{R}^{n} \rightarrow \mathbb{R}$ be a function which is continuous in its second argument and satisfies (25) on $\mathbb{R}_{+} \times \mathbb{R}^{n}$. Assume moreover that

$$
f(s, \pi x)=f(s, x)
$$

for all $(s, x) \in \mathbb{R}_{+} \times \mathbb{R}^{n}$ and each $\pi \in \mathrm{SL}(n)$ induced by a permutation matrix. If

$$
f(s, x)=0 \quad \text { for every }(s, x) \in \mathbb{R}_{+} \times \operatorname{lin}\left\{e_{1}, e_{2}\right\},
$$

then

$$
f(s, x)=0 \quad \text { for every }(s, x) \in \mathbb{R}_{+} \times \mathbb{R}^{n} .
$$

Proof. Let $2 \leq j<n$. Using an induction argument, it suffices to show that

$$
f(s, x)=0 \quad \text { for every }(s, x) \in \mathbb{R}_{+} \times \operatorname{lin}\left\{e_{1}, \ldots, e_{j}\right\}
$$

implies

$$
f(s, x)=0 \quad \text { for every }(s, x) \in \mathbb{R}_{+} \times \operatorname{lin}\left\{e_{1}, \ldots, e_{j+1}\right\} .
$$

Assume that (31) holds. The invariance property (30) then implies

$$
f(s, x)=0 \quad \text { for every }(s, x) \in \mathbb{R}_{+} \times \operatorname{lin}\left\{e_{2}, \ldots, e_{j+1}\right\}
$$

and

$$
f(s, x)=0 \quad \text { for every }(s, x) \in \mathbb{R}_{+} \times \operatorname{lin}\left\{e_{1}, e_{3}, \ldots, e_{j+1}\right\} .
$$

Let $x \in \operatorname{lin}\left\{e_{1}, \ldots, e_{j+1}\right\}$. Suppose that $0<x_{1} / x_{2}<1$ and set $\lambda=x_{1} / x_{2}$. Then

$$
\begin{aligned}
& \left(\psi_{\lambda}^{-t} x\right)_{1}=\left(\phi_{\lambda}^{t} \psi_{\lambda}^{-t} x\right)_{1}=\frac{x_{1}}{1-\lambda}-\frac{\lambda}{1-\lambda} x_{2}=0, \\
& \left(\psi_{\lambda}^{-t} x\right)_{i}=\left(\phi_{\lambda}^{t} \psi_{\lambda}^{-t} x\right)_{i}=0, \quad i=j+2, \ldots, n .
\end{aligned}
$$

Note that (25) gives

$$
f\left(s, \psi_{\lambda}^{-t} x\right)=\lambda^{p} f\left(s \lambda^{q}, \phi_{\lambda}^{t} \psi_{\lambda}^{-t} x\right)+(1-\lambda)^{p} f\left(s(1-\lambda)^{q}, x\right)
$$

By (33) we conclude from this equality that $f\left(s(1-\lambda)^{q}, x\right)=0$ for all $s>0$. Hence (32) holds for $0<x_{1}<x_{2}$ and for $x_{2}<x_{1}<0$.

If $0<\left(x_{1}-x_{2}\right) / x_{1}<1$, then set $\lambda=\left(x_{1}-x_{2}\right) / x_{1}$. Thus

$$
\begin{aligned}
\left(\phi_{\lambda}^{-t} x\right)_{2} & =\left(\psi_{\lambda}^{t} \phi_{\lambda}^{-t} x\right)_{2}=-\frac{1-\lambda}{\lambda} x_{1}+\frac{1}{\lambda} x_{2}=0, \\
\left(\phi_{\lambda}^{-t} x\right)_{i} & =\left(\psi_{\lambda}^{t} \phi_{\lambda}^{-t} x\right)_{i}=0, \quad i=j+2, \ldots, n .
\end{aligned}
$$

Since by (25) we have

$$
f\left(s, \phi_{\lambda}^{-t} x\right)=\lambda^{p} f\left(s \lambda^{q}, x\right)+(1-\lambda)^{p} f\left(s(1-\lambda)^{q}, \psi_{\lambda}^{t} \phi_{\lambda}^{-t} x\right),
$$

relation (34) proves (32) for $0<x_{2}<x_{1}$ and for $x_{1}<x_{2}<0$. 
For $x_{1}, x_{2} \neq 0$ and $\operatorname{sgn}\left(x_{1}\right) \neq \operatorname{sgn}\left(x_{2}\right)$ set $\lambda=x_{1} /\left(x_{1}-x_{2}\right)$. Then $\lambda \in(0,1)$ and

$$
\left(\phi_{\lambda}^{t} x\right)_{2}=\left(\phi_{\lambda}^{t} x\right)_{i}=\left(\psi_{\lambda}^{t} x\right)_{1}=\left(\psi_{\lambda}^{t} x\right)_{i}=0, \quad i=j+2, \ldots, n .
$$

As before we conclude that (32) holds for $x_{1}<0, x_{2}>0$ and for $x_{1}>0, x_{2}<0$. The assumed continuity of $f$ in the second argument concludes the proof.

Next, let us establish a similar result for functions satisfying (26).

Lemma 6. Let $n \geq 3$ and $f: \mathbb{R}_{+} \times \mathbb{R}^{n} \rightarrow \mathbb{R}$ be a function which is continuous in its second argument and satisfies (26) on $\mathbb{R}_{+} \times \mathbb{R}^{n}$. Assume moreover that

$$
f(s, \pi x)=f(s, x)
$$

for all $(s, x) \in \mathbb{R}_{+} \times \mathbb{R}^{n}$ and each $\pi \in \mathrm{SL}(n)$ induced by a permutation matrix. If

$$
f(s, x)=0 \quad \text { for every }(s, x) \in \mathbb{R}_{+} \times \operatorname{lin}\left\{e_{1}, e_{2}\right\},
$$

then

$$
f(s, x)=0 \quad \text { for every }(s, x) \in \mathbb{R}_{+} \times \mathbb{R}^{n} .
$$

Proof. We proceed as in the proof of the previous lemma. Let $2 \leq j<n$. Using an induction argument, it suffices to show that

$$
f(s, x)=0 \quad \text { for every }(s, x) \in \mathbb{R}_{+} \times \operatorname{lin}\left\{e_{1}, \ldots, e_{j}\right\}
$$

implies

$$
f(s, x)=0 \quad \text { for every }(s, x) \in \mathbb{R}_{+} \times \operatorname{lin}\left\{e_{1}, \ldots, e_{j+1}\right\} .
$$

Assume that (36) holds. The invariance property (35) implies

$$
f(s, x)=0 \quad \text { for every }(s, x) \in \mathbb{R}_{+} \times \operatorname{lin}\left\{e_{2}, \ldots, e_{j+1}\right\}
$$

and

$$
f(s, x)=0 \quad \text { for every }(s, x) \in \mathbb{R}_{+} \times \operatorname{lin}\left\{e_{1}, e_{3}, \ldots, e_{j+1}\right\} .
$$

Assume that $x \in \operatorname{lin}\left\{e_{1}, \ldots, e_{j+1}\right\}$. If $x_{1}$ and $x_{2}$ are not both zero and $\operatorname{sgn}\left(x_{1}\right)=\operatorname{sgn}\left(x_{2}\right)$, then $0<x_{2} /\left(x_{1}+x_{2}\right)<1$. Set $\lambda=x_{2} /\left(x_{1}+x_{2}\right)$. Thus

$$
\begin{aligned}
& \left(\phi_{\lambda}^{-1} x\right)_{1}=x_{1}-\frac{1-\lambda}{\lambda} x_{2}=0, \\
& \left(\psi_{\lambda}^{-1} x\right)_{2}=-\frac{\lambda}{1-\lambda} x_{1}+x_{2}=0, \\
& \left(\phi_{\lambda}^{-1} x\right)_{i}=\left(\psi_{\lambda}^{-1} x\right)_{i}=0, \quad i=j+2, \ldots, n .
\end{aligned}
$$

Note that (26) gives

$$
f(s, x)=\lambda^{p} f\left(s \lambda^{q}, \phi_{\lambda}^{-1} x\right)+(1-\lambda)^{p} f\left(s(1-\lambda)^{q}, \psi_{\lambda}^{-1} x\right) .
$$

By (38) and (39) we therefore conclude that $f(s, x)=0$ for all $s>0$. Hence (37) holds for $x_{1}>0, x_{2}>0$ and for $x_{1}<0, x_{2}<0$. 
Suppose that $0<-x_{2} / x_{1}<1$. Let $\lambda=-x_{2} / x_{1}$. Then

$$
\begin{aligned}
\left(\psi_{\lambda} x\right)_{2} & =\left(\phi_{\lambda}^{-1} \psi_{\lambda} x\right)_{2}=0, \\
\left(\psi_{\lambda} x\right)_{i} & =\left(\phi_{\lambda}^{-1} \psi_{\lambda} x\right)_{i}=0, \quad i=j+2, \ldots, n .
\end{aligned}
$$

Note that (26) gives

$$
f\left(s, \psi_{\lambda} x\right)=\lambda^{p} f\left(s \lambda^{q}, \phi_{\lambda}^{-1} \psi_{\lambda} x\right)+(1-\lambda)^{p} f\left(s(1-\lambda)^{q}, x\right) .
$$

By (39) we therefore conclude from this equality that $f\left(s(1-\lambda)^{q}, x\right)=0$ for all $s>0$. Hence (37) holds for $0<-x_{2}<x_{1}$ and for $x_{1}<-x_{2}<0$.

If $0<\left(x_{1}+x_{2}\right) / x_{2}<1$, then set $\lambda=\left(x_{1}+x_{2}\right) / x_{2}$. Thus

$$
\begin{aligned}
\left(\phi_{\lambda} x\right)_{1} & =\left(\psi_{\lambda}^{-1} \phi_{\lambda} x\right)_{1}=0, \\
\left(\phi_{\lambda} x\right)_{i} & =\left(\psi_{\lambda}^{-1} \phi_{\lambda} x\right)_{i}=0, \quad i=j+2, \ldots, n .
\end{aligned}
$$

From (26) and (38) we deduce as before that (37) holds for $0<x_{1}<-x_{2}$ and for $-x_{2}<x_{1}<0$. The continuity of $f$ in the second argument concludes the proof.

\subsection{Representations}

The following result solves the functional equation (24) on a subspace.

Lemma 7. Suppose that $f: \mathbb{R}_{+} \times \mathbb{R}^{n} \rightarrow \mathbb{R}$ is positively homogeneous of degree one in the second argument and satisfies (24) on $\mathbb{R}_{+} \times \mathbb{R}^{n}$. If there exists an $r \in \mathbb{R}$ such that for all $s>0$ and $x \in\left\{ \pm e_{1}, \pm e_{2}\right\}$,

$$
f(s, x)=s^{r} f(1, x),
$$

then for $x_{1}>x_{2}>0$,

$$
\begin{aligned}
f\left(s, x_{1} e_{1}+x_{2} e_{2}\right) & =\left(x_{1}-x_{2}\right)^{-p-r q}\left(x_{1}^{1+p+r q}-x_{2}^{1+p+r q}\right) f\left(s, e_{1}\right), \\
f\left(s,-x_{1} e_{1}-x_{2} e_{2}\right) & =\left(x_{1}-x_{2}\right)^{-p-r q}\left(x_{1}^{1+p+r q}-x_{2}^{1+p+r q}\right) f\left(s,-e_{1}\right) ;
\end{aligned}
$$

for $x_{2}>x_{1}>0$,

$$
\begin{aligned}
f\left(s, x_{1} e_{1}+x_{2} e_{2}\right) & =\left(x_{2}-x_{1}\right)^{-p-r q}\left(x_{2}^{1+p+r q}-x_{1}^{1+p+r q}\right) f\left(s, e_{2}\right), \\
f\left(s,-x_{1} e_{1}-x_{2} e_{2}\right) & =\left(x_{2}-x_{1}\right)^{-p-r q}\left(x_{2}^{1+p+r q}-x_{1}^{1+p+r q}\right) f\left(s,-e_{2}\right) ;
\end{aligned}
$$

and for $x_{1}, x_{2}>0$,

$$
\begin{aligned}
f\left(s,-x_{1} e_{1}+x_{2} e_{2}\right) & =\left(x_{1}+x_{2}\right)^{-p-r q}\left(x_{2}^{1+p+r q} f\left(s, e_{2}\right)+x_{1}^{1+p+r q} f\left(s,-e_{1}\right)\right), \\
f\left(s, x_{1} e_{1}-x_{2} e_{2}\right) & =\left(x_{1}+x_{2}\right)^{-p-r q}\left(x_{1}^{1+p+r q} f\left(s, e_{1}\right)+x_{2}^{1+p+r q} f\left(s,-e_{2}\right)\right) .
\end{aligned}
$$


Proof. Let $\lambda \in(0,1)$. The functional equation (24) evaluated at $e_{1}$ together with the assumed homogeneity of degree one gives

$$
f\left(s, e_{1}\right)=\lambda^{p} f\left(s \lambda^{q}, e_{1}+(1-\lambda) e_{2}\right)+(1-\lambda)^{1+p} f\left(s(1-\lambda)^{q}, e_{1}\right)
$$

for all $s>0$. Let $t>0$. Choose $s=t \lambda^{-q}$ in (41) to arrive at

$$
f\left(t, e_{1}+(1-\lambda) e_{2}\right)=\lambda^{-p}\left[f\left(t \lambda^{-q}, e_{1}\right)-(1-\lambda)^{1+p} f\left(t \lambda^{-q}(1-\lambda)^{q}, e_{1}\right)\right]
$$

for every $t>0$. Thus (40) and equation (42) evaluated at $t=1$ yield

$$
\begin{aligned}
f\left(t, e_{1}+(1-\lambda) e_{2}\right) & =t^{r} \lambda^{-p}\left[f\left(\lambda^{-q}, e_{1}\right)-(1-\lambda)^{1+p} f\left(\lambda^{-q}(1-\lambda)^{q}, e_{1}\right)\right] \\
& =t^{r} f\left(1, e_{1}+(1-\lambda) e_{2}\right) .
\end{aligned}
$$

This and (40) show that we can rewrite (41) as

$$
f\left(s, e_{1}+(1-\lambda) e_{2}\right)=\lambda^{-p-r q}\left(1-(1-\lambda)^{1+p+r q}\right) f\left(s, e_{1}\right) .
$$

Let $x_{1}>x_{2}>0$ and set $\lambda=1-x_{2} / x_{1}$. Since $f$ is homogeneous of degree one in the second component we obtain

$$
\begin{aligned}
f\left(s, x_{1} e_{1}+x_{2} e_{2}\right) & =x_{1} f\left(s, e_{1}+(1-\lambda) e_{2}\right) \\
& =x_{1}\left(1-x_{2} / x_{1}\right)^{-p-r q}\left(1-\left(x_{2} / x_{1}\right)^{1+p+r q}\right) f\left(s, e_{1}\right) \\
& =\left(x_{1}-x_{2}\right)^{-p-r q}\left(x_{1}^{1+p+r q}-x_{2}^{1+p+r q}\right) f\left(s, e_{1}\right) .
\end{aligned}
$$

Replacing $e_{1}$ and $e_{2}$ by $-e_{1}$ and $-e_{2}$, respectively, in the above derivation shows

$$
f\left(s,-x_{1} e_{1}-x_{2} e_{2}\right)=\left(x_{1}-x_{2}\right)^{-p-r q}\left(x_{1}^{1+p+r q}-x_{2}^{1+p+r q}\right) f\left(s,-e_{1}\right)
$$

for $x_{1}>x_{2}>0$.

Let $0<\lambda<1$. The functional equation (24) evaluated at $e_{2}$ together with the assumed homogeneity of degree one gives

$$
f\left(s, e_{2}\right)=\lambda^{1+p} f\left(s \lambda^{q}, e_{2}\right)+(1-\lambda)^{p} f\left(s(1-\lambda)^{q}, \lambda e_{1}+e_{2}\right)
$$

for all $s>0$. Let $t>0$. Choose $s=t(1-\lambda)^{-q}$ in (44) to get

$$
f\left(t, \lambda e_{1}+e_{2}\right)=(1-\lambda)^{-p}\left[f\left(t(1-\lambda)^{-q}, e_{2}\right)-\lambda^{1+p} f\left(t \lambda^{q}(1-\lambda)^{-q}, e_{2}\right)\right]
$$

for every $t>0$. Thus (40) and equation (45) evaluated at $t=1$ yield

$$
\begin{aligned}
f\left(t, \lambda e_{1}+e_{2}\right) & =t^{r}(1-\lambda)^{-p}\left[f\left((1-\lambda)^{-q}, e_{2}\right)-\lambda^{1+p} f\left(\lambda^{q}(1-\lambda)^{-q}, e_{2}\right)\right] \\
& =t^{r} f\left(1, \lambda e_{1}+e_{2}\right) .
\end{aligned}
$$

This and (40) show that we can rewrite (44) as

$$
f\left(s, \lambda e_{1}+e_{2}\right)=(1-\lambda)^{-p-r q}\left(1-\lambda^{1+p+r q}\right) f\left(s, e_{2}\right) .
$$


Let $x_{2}>x_{1}>0$ and set $\lambda=x_{1} / x_{2}$. By homogeneity we obtain

$$
\begin{aligned}
f\left(s, x_{1} e_{1}+x_{2} e_{2}\right) & =x_{2} f\left(s, \lambda e_{1}+e_{2}\right) \\
& =x_{2}\left(1-x_{1} / x_{2}\right)^{-p-r q}\left(1-\left(x_{1} / x_{2}\right)^{1+p+r q}\right) f\left(s, e_{2}\right) \\
& =\left(x_{2}-x_{1}\right)^{-p-r q}\left(x_{2}^{1+p+r q}-x_{1}^{1+p+r q}\right) f\left(s, e_{2}\right) .
\end{aligned}
$$

Replacing $e_{1}$ and $e_{2}$ by $-e_{1}$ and $-e_{2}$, respectively, in the above derivation shows

$$
f\left(s,-x_{1} e_{1}-x_{2} e_{2}\right)=\left(x_{2}-x_{1}\right)^{-p-r q}\left(x_{2}^{1+p+r q}-x_{1}^{1+p+r q}\right) f\left(s,-e_{2}\right)
$$

for $x_{2}>x_{1}>0$.

Let $\lambda \in(0,1)$. The functional equation (24) evaluated at $-\lambda e_{1}+(1-\lambda) e_{2}$ together with the homogeneity of degree one gives

$$
f\left(s,-\lambda e_{1}+(1-\lambda) e_{2}\right)=\lambda^{1+p+r q} f\left(s,-e_{1}\right)+(1-\lambda)^{1+p+r q} f\left(s, e_{2}\right) .
$$

Suppose that both $x_{1}$ and $x_{2}$ are positive and set $\lambda=x_{1} /\left(x_{1}+x_{2}\right)$. By homogeneity,

$$
\begin{aligned}
f\left(s,-x_{1} e_{1}+x_{2} e_{2}\right) & =\left(x_{1}+x_{2}\right) f\left(s,-\lambda e_{1}+(1-\lambda) e_{2}\right) \\
& =\left(x_{1}+x_{2}\right)^{-p-r q}\left(x_{2}^{1+p+r q} f\left(s, e_{2}\right)+x_{1}^{1+p+r q} f\left(s,-e_{1}\right)\right) .
\end{aligned}
$$

Replacing $e_{1}$ and $e_{2}$ by $-e_{1}$ and $-e_{2}$, respectively, in the above derivation shows

$$
f\left(s, x_{1} e_{1}-x_{2} e_{2}\right)=\left(x_{1}+x_{2}\right)^{-p-r q}\left(x_{1}^{1+p+r q} f\left(s, e_{1}\right)+x_{2}^{1+p+r q} f\left(s,-e_{2}\right)\right) .
$$

\section{The contravariant case}

\subsection{Preliminaries}

Let us first show that $\operatorname{SL}(n)$ contravariant operators map $(n-1)$-dimensional simplices to line segments.

Lemma 8. Let $n \geq 3$, let $\mathrm{Z}: \mathcal{P}_{o}^{n} \rightarrow \mathcal{K}^{n}$ be $\mathrm{SL}(n)$ contravariant, and set $T^{\prime}=T^{n} \cap e_{1}^{\perp}$. Then there exists a non-negative constant a with

$$
\mathrm{Z}\left(s T^{\prime}\right)=a s^{n-1}\left[-e_{1}, e_{1}\right] \text { for every } s>0 .
$$

Proof. For arbitrary $s>0$ and $2 \leq k \leq n$, let $\phi \in \operatorname{SL}(n)$ be the map defined by

$$
\phi e_{1}=e_{1}+s e_{k} \quad \text { and } \phi e_{i}=e_{i}, \quad i=2, \ldots n .
$$

Note that for $x \in \mathbb{R}^{n}$ we have $\left(\phi^{-t} x\right)_{1}=x_{1}-s x_{k}$. Clearly, $\phi T^{\prime}=T^{\prime}$. If $x \in Z T^{\prime}$, then the contravariance of $\mathrm{Z}$ implies that also $\phi^{-t} x \in \mathrm{Z} T^{\prime}$. Since $\mathrm{Z} T^{\prime}$ is bounded, we conclude that $x_{2}=\cdots=x_{n}=0$. Thus there exist $a, b \in \mathbb{R}$ such that $-a \leq b$ and $\mathrm{Z} T^{\prime}=\left[-a e_{1}, b e_{1}\right]$. Define $\psi \in \mathrm{SL}(n)$ by

$$
\psi e_{1}=-e_{1}, \quad \psi e_{2}=e_{3}, \quad \psi e_{3}=e_{2}, \quad \psi e_{i}=e_{i}, \quad i=4, \ldots, n \text { if } n>3 .
$$


Since $\psi T^{\prime}=T^{\prime}$, the $\mathrm{SL}(n)$ contravariance of $\mathrm{Z}$ implies

$$
\left[-a e_{1}, b e_{1}\right]=\mathrm{Z} T^{\prime}=\psi^{-t} \mathrm{Z} T^{\prime}=\left[-b e_{1}, a e_{1}\right]
$$

and hence $a=b$. Finally, for $s>0$, define $\tau \in \operatorname{SL}(n)$ by

$$
\tau e_{1}=s^{1-n} e_{1}, \quad \tau e_{i}=s e_{i}, \quad i=2, \ldots, n
$$

Note that $\tau T^{\prime}=s T^{\prime}$ and $\tau^{-t}\left(a\left[-e_{1}, e_{1}\right]\right)=a s^{n-1}\left[-e_{1}, e_{1}\right]$. The SL $(n)$ contravariance of $\mathrm{Z}$ therefore concludes the proof of the lemma.

Assume that $\mathrm{Z}: \mathcal{P}_{o}^{n} \rightarrow\left\langle\mathcal{K}^{n},+\right\rangle$ is an $\mathrm{SL}(n)$ contravariant Minkowski valuation and let $\lambda \in(0,1)$. Now, we are going to derive a functional equation for the support function of $\mathrm{Z}$. This equation is closely related to those treated in Section 5 and will be frequently used. Denote by $H_{\lambda}$ the hyperplane through $o$ with normal vector $\lambda e_{1}-(1-\lambda) e_{2}$ and set $T^{\prime}=T^{n} \cap e_{1}^{\perp}$. Note that

$$
\left(s T^{n}\right) \cap H_{\lambda}^{+}=s \phi_{\lambda} T^{n}, \quad\left(s T^{n}\right) \cap H_{\lambda}^{-}=s \psi_{\lambda} T^{n} \quad \text { and } \quad\left(s T^{n}\right) \cap H_{\lambda}=s \phi_{\lambda} T^{\prime} .
$$

So the valuation property of $\mathrm{Z}$ implies

$$
\mathrm{Z}\left(s T^{n}\right)+\mathrm{Z}\left(s \phi_{\lambda} T^{\prime}\right)=\mathrm{Z}\left(s \phi_{\lambda} T^{n}\right)+\mathrm{Z}\left(s \psi_{\lambda} T^{n}\right)
$$

The $\operatorname{SL}(n)$ contravariance of $\mathrm{Z}$ therefore gives

$$
\begin{aligned}
& \mathrm{Z}\left(s T^{n}\right)+\lambda^{1 / n} \phi_{\lambda}^{-t} \mathrm{Z}\left(s \lambda^{1 / n} T^{\prime}\right) \\
& \quad=\lambda^{1 / n} \phi_{\lambda}^{-t} \mathrm{Z}\left(s \lambda^{1 / n} T^{n}\right)+(1-\lambda)^{1 / n} \psi_{\lambda}^{-t} \mathrm{Z}\left(s(1-\lambda)^{1 / n} T^{n}\right) .
\end{aligned}
$$

Let $\rho \in \operatorname{SL}(n)$ be defined by $\rho e_{1}=e_{2}, \rho e_{2}=-e_{1}$, and $\rho e_{k}=e_{k}$ for $k \geq 3$. Hence $\rho$ is the counterclockwise rotation by an angle of $\pi / 2$ in the plane spanned by the first two canonical basis vectors. For $g(\lambda)=\lambda$ and $h(\lambda)=1-\lambda$ we obtain

$$
\rho \phi_{\lambda}^{-t} \rho^{-1}=\lambda^{-1} \phi_{\lambda, g} \quad \text { and } \quad \rho \psi_{\lambda}^{-t} \rho^{-1}=(1-\lambda)^{-1} \psi_{\lambda, h}
$$

Let $\hat{\mathrm{Z}}: \mathcal{P}_{o}^{n} \rightarrow \mathcal{K}^{n}$ be defined by $\hat{\mathrm{Z}} K=\rho \mathrm{Z} K$. By (49) we deduce

$$
\begin{aligned}
\hat{\mathrm{Z}}\left(s T^{n}\right)+\lambda^{1 / n-1} \phi_{\lambda, g} \hat{\mathrm{Z}}\left(s \lambda^{1 / n} T^{\prime}\right) & \\
& =\lambda^{1 / n-1} \phi_{\lambda, g} \hat{\mathrm{Z}}\left(s \lambda^{1 / n} T^{n}\right)+(1-\lambda)^{1 / n-1} \psi_{\lambda, h} \hat{\mathrm{Z}}\left(s(1-\lambda)^{1 / n} T^{n}\right) .
\end{aligned}
$$

After these preparations we are in a position to prove our first main result. 


\subsection{The crucial classification}

In this subsection we establish a theorem which has all the main results on contravariant valuations stated in Sections 1 and 3 as consequences.

Theorem 7. Let $n \geq 3$. Suppose that $\mathrm{Z}: \mathcal{P}_{o}^{n} \rightarrow\left\langle\mathcal{K}^{n},+\right\rangle$ is an $\operatorname{SL}(n)$ contravariant Minkowski valuation such that the two functions

$$
s \mapsto h_{\mathrm{Z}\left(s T^{n}\right)}\left(e_{3}\right) \quad \text { and } \quad s \mapsto h_{\mathrm{Z}\left(s T^{n}\right)}\left(-e_{3}\right), \quad s>0,
$$

are bounded from below on some non-empty open intervals $I_{+} \subset \mathbb{R}_{+}$and $I_{-} \subset \mathbb{R}_{+}$, respectively. Then there exist constants $c_{1}, c_{2}, c_{3}$ with $c_{1} \geq 0$ and $c_{1}+c_{2}+c_{3} \geq 0$ such that

$$
h_{\mathrm{Z} P}=c_{1} h_{\Pi P}+c_{2} h_{\Pi_{o} P}+c_{3} h_{\Pi_{o}(-P)} \quad \text { for every } P \in \mathcal{P}_{o}^{n} .
$$

Proof. Define a function $f: \mathbb{R}_{+} \times \mathbb{R}^{n} \rightarrow \mathbb{R}$ by

$$
f(s, x)=h_{\hat{\mathrm{Z}}\left(s T^{n}\right)}(x)-h_{\hat{\mathrm{Z}}\left(s T^{\prime}\right)}(x),
$$

where as before $\hat{\mathrm{Z}} K=\rho \mathrm{Z} K$ and $T^{\prime}=T^{n} \cap e_{1}^{\perp}$. Obviously we have $\psi_{\lambda} T^{\prime}=T^{\prime}$. By (50) this implies that

$$
(1-\lambda)^{1 / n-1} \psi_{\lambda, h} \hat{\mathrm{Z}}\left(s(1-\lambda)^{1 / n} T^{\prime}\right)=\hat{\mathrm{Z}}\left(s T^{\prime}\right) .
$$

Hence we infer from (51) that $f$ satisfies (24) for $p=1 / n-1$ and $q=1 / n$, i.e.

$$
f(s, x)=\lambda^{1 / n-1} f\left(s \lambda^{1 / n}, \phi_{\lambda, g}^{t} x\right)+(1-\lambda)^{1 / n-1} f\left(s(1-\lambda)^{1 / n}, \psi_{\lambda, h}^{t} x\right) .
$$

But $f$ is positively homogeneous of degree one in $x$, and hence

$$
f\left(s, \pm e_{3}\right)=\lambda^{1 / n} f\left(s \lambda^{1 / n}, \pm \phi_{\lambda}^{t} e_{3}\right)+(1-\lambda)^{1 / n} f\left(s(1-\lambda)^{1 / n}, \pm \psi_{\lambda}^{t} e_{3}\right) .
$$

In other words, the function $f$ satisfies (25) at the points $\pm e_{3}$. Let $s>0$. By Lemma 8 there exists a non-negative constant $a$ such that

$$
h_{\hat{\mathrm{Z}}\left(s T^{\prime}\right)}(x)=a s^{n-1}\left|x_{2}\right|, \quad s>0 .
$$

This and the assumed boundedness of the functions $s \mapsto h_{\mathrm{Z}\left(s T^{n}\right)}\left( \pm e_{3}\right)$ show that $f\left(\cdot, e_{3}\right)$ and $f\left(\cdot,-e_{3}\right)$ are bounded from below on some non-empty open intervals $I_{+} \subset \mathbb{R}_{+}$and $I_{-} \subset \mathbb{R}_{+}$, respectively. So Lemma 4 implies

$$
f\left(s, e_{3}\right)=s^{n-1} f\left(1, e_{3}\right) \text { and } f\left(s,-e_{3}\right)=s^{n-1} f\left(1,-e_{3}\right) .
$$

Since $h_{\hat{\mathrm{Z}}\left(s T^{\prime}\right)}\left(e_{3}\right)=0$ by $(52)$, we obtain

$$
h_{\hat{\mathrm{Z}}\left(s T^{n}\right)}\left(e_{3}\right)=f\left(s, e_{3}\right)=s^{n-1} f\left(1, e_{3}\right)=s^{n-1} h_{\hat{\mathrm{Z}} T^{n}}\left(e_{3}\right) .
$$

Similarly, we deduce that $h_{\hat{\mathrm{Z}}\left(s T^{n}\right)}\left(-e_{3}\right)=s^{n-1} h_{\hat{\mathrm{Z}} T^{n}}\left(-e_{3}\right)$. Since both $e_{3}$ and $-e_{3}$ are fixpoints of $\rho$, we infer from (5) that

$$
h_{\mathrm{Z}\left(s T^{n}\right)}\left(e_{3}\right)=s^{n-1} h_{\mathrm{Z} T^{n}}\left(e_{3}\right) \quad \text { and } \quad h_{\mathrm{Z}\left(s T^{n}\right)}\left(-e_{3}\right)=s^{n-1} h_{\mathrm{Z} T^{n}}\left(-e_{3}\right) .
$$


The $\mathrm{SL}(n)$ contravariance of $\mathrm{Z}$ proves

$$
h_{\mathrm{Z}\left(s T^{n}\right)}(\pi x)=h_{\mathrm{Z}\left(s T^{n}\right)}(x), \quad x \in \mathbb{R}^{n},
$$

for every map $\pi \in \operatorname{SL}(n)$ which is induced by a permutation matrix. Hence

$$
h_{\mathrm{Z}\left(s T^{n}\right)}(x)=s^{n-1} h_{\mathrm{Z} T^{n}}(x) \quad \text { for every } x \in\left\{ \pm e_{1}, \pm e_{2}\right\} .
$$

Since $\rho^{t}\left\{ \pm e_{1}, \pm e_{2}\right\}=\left\{ \pm e_{1}, \pm e_{2}\right\}$, we infer from (5) that for every $x \in\left\{ \pm e_{1}, \pm e_{2}\right\}$,

$$
h_{\hat{\mathrm{Z}}\left(s T^{n}\right)}(x)=h_{\mathrm{Z}\left(s T^{n}\right)}\left(\rho^{t} x\right)=s^{n-1} h_{\mathrm{Z} T^{n}}\left(\rho^{t} x\right)=s^{n-1} h_{\hat{\mathrm{Z}} T^{n}}(x) .
$$

The equality (52) therefore implies

$$
f\left(s, e_{2}\right)=h_{\hat{\mathrm{Z}}\left(s T^{n}\right)}\left(e_{2}\right)-a s^{n-1}=s^{n-1}\left[h_{\hat{\mathrm{Z}} T^{n}}\left(e_{2}\right)-a\right]=s^{n-1} f\left(1, e_{2}\right) .
$$

Replacing $e_{2}$ by $-e_{2}$ in the above derivation proves $f\left(s,-e_{2}\right)=s^{n-1} f\left(1,-e_{2}\right)$. Moreover, since $f\left(s, \pm e_{1}\right)=h_{\hat{\mathrm{Z}}\left(s T^{n}\right)}\left( \pm e_{1}\right)$ by (52), we deduce from (54) that $f\left(s, e_{1}\right)=$ $s^{n-1} f\left(1, e_{1}\right)$ and $f\left(s,-e_{1}\right)=s^{n-1} f\left(1,-e_{1}\right)$. An application of Lemma 7 for $p=$ $1 / n-1, q=1 / n$, and $r=n-1$ shows that for $x_{1}>x_{2}>0$,

$$
\begin{aligned}
f\left(s, x_{1} e_{1}+x_{2} e_{2}\right) & =\left(x_{1}-x_{2}\right) f\left(s, e_{1}\right), \\
f\left(s,-x_{1} e_{1}-x_{2} e_{2}\right) & =\left(x_{1}-x_{2}\right) f\left(s,-e_{1}\right) ;
\end{aligned}
$$

for $x_{2}>x_{1}>0$,

$$
\begin{aligned}
f\left(s, x_{1} e_{1}+x_{2} e_{2}\right) & =\left(x_{2}-x_{1}\right) f\left(s, e_{2}\right), \\
f\left(s,-x_{1} e_{1}-x_{2} e_{2}\right) & =\left(x_{2}-x_{1}\right) f\left(s,-e_{2}\right) ;
\end{aligned}
$$

and for $x_{1}, x_{2}>0$,

$$
\begin{aligned}
f\left(s,-x_{1} e_{1}+x_{2} e_{2}\right) & =x_{2} f\left(s, e_{2}\right)+x_{1} f\left(s,-e_{1}\right), \\
f\left(s, x_{1} e_{1}-x_{2} e_{2}\right) & =x_{1} f\left(s, e_{1}\right)+x_{2} f\left(s,-e_{2}\right) .
\end{aligned}
$$

The positive homogeneity of the functions $f\left(\cdot, \pm e_{i}\right), i=1,2$, and the fact that

$$
h_{\mathrm{Z}\left(s T^{n}\right)}\left(x_{1} e_{1}+x_{2} e_{2}\right)=f\left(s,-x_{2} e_{1}+x_{1} e_{2}\right)+a s^{n-1}\left|x_{1}\right|
$$

proves, for $x_{1}>x_{2}>0$,

$$
\begin{aligned}
h_{\mathrm{Z}\left(s T^{n}\right)}\left(x_{1} e_{1}-x_{2} e_{2}\right) & =s^{n-1}\left[x_{1}\left(f\left(1, e_{2}\right)+a\right)-x_{2} f\left(1, e_{2}\right)\right], \\
h_{\mathrm{Z}\left(s T^{n}\right)}\left(-x_{1} e_{1}+x_{2} e_{2}\right) & =s^{n-1}\left[x_{1}\left(f\left(1,-e_{2}\right)+a\right)-x_{2} f\left(1,-e_{2}\right)\right]
\end{aligned}
$$

for $x_{2}>x_{1}>0$,

$$
\begin{aligned}
h_{\mathrm{Z}\left(s T^{n}\right)}\left(x_{1} e_{1}-x_{2} e_{2}\right) & =s^{n-1}\left[x_{1}\left(a-f\left(1, e_{1}\right)\right)+x_{2} f\left(1, e_{1}\right)\right], \\
h_{\mathrm{Z}\left(s T^{n}\right)}\left(-x_{1} e_{1}+x_{2} e_{2}\right) & =s^{n-1}\left[x_{1}\left(a-f\left(1,-e_{1}\right)\right)+x_{2} f\left(1,-e_{1}\right)\right] ;
\end{aligned}
$$


and for $x_{1}, x_{2}>0$,

$$
\begin{aligned}
h_{\mathrm{Z}\left(s T^{n}\right)}\left(x_{1} e_{1}+x_{2} e_{2}\right) & =s^{n-1}\left[x_{1}\left(a+f\left(1, e_{2}\right)\right)+x_{2} f\left(1,-e_{1}\right)\right], \\
h_{\mathrm{Z}\left(s T^{n}\right)}\left(-x_{1} e_{1}-x_{2} e_{2}\right) & =s^{n-1}\left[x_{1}\left(a+f\left(1,-e_{2}\right)\right)+x_{2} f\left(1, e_{1}\right)\right] .
\end{aligned}
$$

Define a map $\phi \in \operatorname{SL}(n)$ with

$$
\phi e_{1}=e_{3}, \quad \phi e_{2}=e_{1}, \quad \phi e_{3}=e_{2}, \quad \phi e_{i}=e_{i}, \quad i=4, \ldots, n \text { if } n>3 .
$$

Then $\phi T^{n}=T^{n}$, and the contravariance of $\mathrm{Z}$ proves $h_{\mathrm{Z} T^{n}}\left(e_{1}\right)=h_{\mathrm{Z} T^{n}}\left(e_{2}\right)$ as well as $h_{\mathrm{Z} T^{n}}\left(-e_{1}\right)=h_{\mathrm{Z} T^{n}}\left(-e_{2}\right)$. Thus by the definition of $f,(52)$, the definition of $\hat{Z}$, and (5) we arrive at

$$
\begin{aligned}
f\left(1, e_{1}\right) & =h_{\hat{\mathrm{Z}} T^{n}}\left(e_{1}\right)=h_{\mathrm{Z} T^{n}}\left(\rho^{t} e_{1}\right)=h_{\mathrm{Z} T^{n}}\left(-e_{2}\right)=h_{\mathrm{Z} T^{n}}\left(-e_{1}\right)=h_{\hat{\mathrm{Z}} T^{n}}\left(-e_{2}\right) \\
& =f\left(1,-e_{2}\right)+a .
\end{aligned}
$$

Similarly, by replacing $e_{1}$ and $e_{2}$ by $-e_{1}$ and $-e_{2}$, respectively, in the above derivation one sees that $f\left(1,-e_{1}\right)=f\left(1, e_{2}\right)+a$. Define constants

$$
\begin{aligned}
& c_{1}=(n-1) !\left[f\left(1, e_{2}\right)+f\left(1,-e_{2}\right)+a\right], \\
& c_{2}=-(n-1) ! f\left(1, e_{2}\right), \\
& c_{3}=-(n-1) ! f\left(1,-e_{2}\right) .
\end{aligned}
$$

Using formulas (14) and (15) as well as the positive homogeneity of degree $n-1$ of the operators $\Pi$ and $\Pi_{o}$ we see that

$h_{\mathrm{Z}\left(s T^{n}\right)}(x)=c_{1} h_{\Pi\left(s T^{n}\right)}(x)+c_{2} h_{\Pi_{o}\left(s T^{n}\right)}(x)+c_{3} h_{\Pi_{o}\left(-s T^{n}\right)}(x), \quad x \in \operatorname{lin}\left\{e_{1}, e_{2}\right\}$.

For all $s>0$ and $x \in \mathbb{R}^{n}$ we have

$$
h_{\mathrm{Z}\left(s T^{\prime}\right)}(x)=a s^{n-1}\left|x_{1}\right|=c_{1} h_{\Pi\left(s T^{\prime}\right)}(x)+c_{2} h_{\Pi_{o}\left(s T^{\prime}\right)}(x)+c_{3} h_{\Pi_{o}\left(-s T^{\prime}\right)}(x) .
$$

Hence, by (49), the function $g: \mathbb{R}_{+} \times \mathbb{R}^{n} \rightarrow \mathbb{R}$ defined by

$$
g(s, x)=h_{\mathrm{Z}\left(s T^{n}\right)}(x)-c_{1} h_{\Pi\left(s T^{n}\right)}(x)-c_{2} h_{\Pi_{o}\left(s T^{n}\right)}(x)-c_{3} h_{\Pi_{o}\left(-s T^{n}\right)}(x)
$$

satisfies (26). From (53), (55), and the $\operatorname{SL}(n)$ contravariance of the operators $\Pi$ and $\Pi_{o}$ we see that $g$ satisfies the conditions of Lemma 6 . Thus the equality (55) actually holds for all $x \in \mathbb{R}^{n}$. Lemma 3 implies that

$$
h_{\mathrm{Z} P}=c_{1} h_{\Pi P}+c_{2} h_{\Pi_{o} P}+c_{3} h_{\Pi_{o}(-P)} \quad \text { for all } P \in \mathcal{P}_{o}^{n} .
$$

Note that by the definition of the constants $c_{1}, c_{2}$, and $c_{3}$ and the non-negativity of $a$ we have $c_{1}+c_{2}+c_{3}=a(n-1) ! \geq 0$. Let $P \in \mathcal{P}_{o}^{n}$ be chosen such that the origin is an interior point of $P$. Thus $h_{\mathrm{Z} P}=c_{1} h_{\Pi P}$ and we conclude that $c_{1} h_{\Pi P}$ is a support function. From (3) we infer

$$
0 \leq c_{1}\left[h_{\Pi P}(x)+h_{\Pi P}(y)-h_{\Pi P}(x+y)\right] \quad \text { for all } x, y \in \mathbb{R}^{n} .
$$

In particular, if we take $P=[-1,1]^{n}, x=e_{1}$, and $y=-e_{1}$, then we get $c_{1} \geq 0$. 


\subsection{Proofs of the main theorems}

Using the previous result, we are now in a position to establish all theorems on $\operatorname{SL}(n)$ contravariant Minkowski valuations stated in Sections 1 and 3.

Proof of Theorem 4. Assume that Z: $\mathcal{K}_{o}^{n} \rightarrow \mathcal{K}^{n}$ is a continuous $\operatorname{SL}(n)$ contravariant Minkowski valuation. The continuity of $\mathrm{Z}$ implies that the two functions

$$
s \mapsto h_{\mathrm{Z}\left(s T^{n}\right)}\left(e_{3}\right) \quad \text { and } \quad s \mapsto h_{\mathrm{Z}\left(s T^{n}\right)}\left(-e_{3}\right), \quad s>0,
$$

are continuous. Hence they are bounded from below on some non-empty open intervals $I_{+} \subset \mathbb{R}_{+}$and $I_{-} \subset \mathbb{R}_{+}$, respectively. Theorem 7 shows that there exist a non-negative constant $c_{1}$ as well as constants $c_{2}, c_{3} \in \mathbb{R}$ with

$$
h_{\mathrm{Z} P}=c_{1} h_{\Pi P}+c_{2} h_{\Pi_{o} P}+c_{3} h_{\Pi_{o}(-P)} \quad \text { for every } P \in \mathcal{P}_{o}^{n} .
$$

Since $\mathrm{Z}$ and $\Pi$ are continuous but $\Pi_{o}$ is not continuous at polytopes containing the origin on their boundaries, we have $h_{\mathrm{Z} P}=c_{1} h_{\Pi P}$ for every $P \in \mathcal{P}_{o}^{n}$. The continuity of $\mathrm{Z}$ and $\Pi$ as well as the fact that $\mathcal{P}_{o}^{n}$ is a dense subset of $\mathcal{K}_{o}^{n}$ prove $h_{\mathrm{Z} K}=c_{1} h_{\Pi K}$ for every $K \in \mathcal{K}_{o}^{n}$.

Proof of Theorem 3. Let $s>0$. Since the origin is contained in $\mathrm{Z}\left(s T^{n}\right)$, we have $h_{\mathrm{Z}\left(s T^{n}\right)} \geq 0$. Hence the functions $h_{\mathrm{Z}\left(s T^{n}\right)}\left( \pm e_{3}\right)$ are bounded from below on $\mathbb{R}_{+}$. By Theorem 7 there exist a non-negative constant $c_{1}$ as well as constants $c_{2}, c_{3} \in \mathbb{R}$ with $c_{1}+c_{2}+c_{3} \geq 0$ and

$$
h_{\mathrm{Z} P}=c_{1} h_{\Pi P}+c_{2} h_{\Pi_{o} P}+c_{3} h_{\Pi_{o}(-P)} \quad \text { for every } P \in \mathcal{P}_{o}^{n} .
$$

Proof of Theorem 1. Theorem 1 is an immediate consequence of Theorem 4.

\section{The covariant case}

\subsection{Preliminaries}

Let us collect some basic facts about covariant operators. The following statement is an immediate consequence of the definition of covariance and (5).

Lemma 9. Let $n \geq 3,1 \leq k<n$. For $i=1, \ldots$, m let $c_{i} \in \mathbb{R}$ and suppose that $\mathrm{Z}_{i}$ : $\mathcal{P}_{o}^{n} \rightarrow\left\langle\mathcal{K}^{n},+\right\rangle$ are $\mathrm{SL}(n)$ covariant maps. If $\sum_{i=1}^{m} c_{i} h_{\mathrm{Z}_{i} P}=0$ for every $k$-dimensional convex polytope $P \in \mathcal{P}_{o}^{n}$ which is contained in $\operatorname{lin}\left\{e_{1}, \ldots, e_{k}\right\}$, then $\sum_{i=1}^{m} c_{i} h_{\mathrm{Z}_{i} P}=0$ for every $k$-dimensional convex polytope $P \in \mathcal{P}_{o}^{n}$.

The next lemma is due to Ludwig [38]. For completeness we include a proof.

Lemma 10. Let $n \geq 3$. If $\mathrm{Z}: \mathcal{P}_{o}^{n} \rightarrow \mathcal{K}^{n}$ is $\mathrm{SL}(n)$ covariant, then $\mathrm{Z} P \subset \operatorname{lin} P$ for every $P \in \mathcal{P}_{o}^{n}$. 
Proof. The statement is trivial if $P$ is $n$-dimensional. Next, assume that $\operatorname{dim} P=0$. Hence $P=\{o\}$. Define $\phi \in \operatorname{SL}(n)$ by

$$
\phi e_{1}=2 e_{1}, \quad \phi e_{2}=2^{-1} e_{2}, \quad \phi e_{k}=e_{k}, \quad 3 \leq k \leq n .
$$

Clearly, $\phi P=P$ and so the covariance of $\mathrm{Z}$ together with (5) and the fact that support functions are positively homogeneous of degree one yields

$$
h_{\mathrm{Z} P}\left(e_{1}\right)=h_{\mathrm{Z} \phi P}\left(e_{1}\right)=h_{\phi \mathrm{Z} P}\left(e_{1}\right)=h_{\mathrm{Z} P}\left(\phi^{t} e_{1}\right)=2 h_{\mathrm{Z} P}\left(e_{1}\right) .
$$

Hence $h_{\mathrm{Z} P}\left(e_{1}\right)=0$. For each unit vector $u \in S^{n-1}$ it is possible to find a rotation $\vartheta \in \operatorname{SL}(n)$ such that $u=\vartheta e_{1}$. This, (5), the covariance of $\mathrm{Z}$, and the obvious equality $\vartheta^{t} P=P$ imply

$$
h_{\mathrm{Z} P}(u)=h_{\mathrm{Z} P}\left(\vartheta e_{1}\right)=h_{\vartheta^{t} \mathrm{Z} P}\left(e_{1}\right)=h_{\mathrm{Z} \vartheta^{t} P}\left(e_{1}\right)=h_{\mathrm{Z} P}\left(e_{1}\right)=0 .
$$

By homogeneity we conclude that $h_{\mathrm{Z} P}=0$. This proves $\mathrm{Z} P=\{o\}$ and therefore settles the 0-dimensional case.

Finally, assume that $0<\operatorname{dim} P=k<n$ and, without loss of generality, that $P \subset$ $\operatorname{lin}\left\{e_{1}, \ldots, e_{k}\right\}$. For arbitrary $s>0$, let $\phi \in \operatorname{SL}(n)$ be defined by

$$
\phi e_{i}=e_{i}, \quad i=1, \ldots, k, \quad \text { and } \phi e_{i}=s e_{i-k}+e_{i}, \quad i=k+1, \ldots, n .
$$

Clearly, $\phi P=P$. If $x \in \mathrm{Z} P$, then the covariance of $\mathrm{Z}$ implies that also $\phi x \in \mathrm{Z} P$. Since $\mathrm{Z} P$ is bounded and $s$ can be arbitrarily large we conclude that $x_{k+1}=\cdots=x_{n}=0$.

Corollary 2. Suppose that $n \geq 3$ and $\mathrm{Z}: \mathcal{P}_{o}^{n} \rightarrow \mathcal{K}^{n}$ is $\mathrm{SL}(n)$ covariant. If $0<k<n$ and $P \in \operatorname{lin}\left\{e_{1}, \ldots, e_{k}\right\}$, then

$$
\mathrm{Z}(s P)=s \mathrm{Z} P \quad \text { for any } s>0 .
$$

If $1<k<n$ and $P \subset \operatorname{lin}\left\{e_{1}, \ldots, e_{k}\right\}$, then

$$
\mathrm{Z}\left(\phi_{\lambda} P\right)=\phi_{\lambda} \mathrm{Z} P \quad \text { and } \quad \mathrm{Z}\left(\psi_{\lambda} P\right)=\psi_{\lambda} \mathrm{Z} P \quad \text { for all } \lambda \in(0,1) .
$$

Proof. For $s>0$ define $\phi \in \operatorname{SL}(n)$ by

$$
\phi e_{i}=s e_{i}, \quad i=1, \ldots, n-1, \quad \text { and } \phi e_{n}=s^{1-n} e_{n} .
$$

Clearly, $\phi P=s P$. Lemma 10 shows that also $\phi \mathrm{Z} P=s \mathrm{Z} P$. From the $\mathrm{SL}(n)$ covariance of $\mathrm{Z}$ we deduce (57).

The SL $(n)$ covariance of $\mathrm{Z}$ together with (57) proves that $\mathrm{Z}(\phi P)=\phi \mathrm{Z} P$ for every $P \subset \operatorname{lin}\left\{e_{1}, \ldots, e_{k}\right\}$ and all invertible linear transformations $\phi$ with positive determinant which fix $\operatorname{lin}\left\{e_{1}, \ldots, e_{k}\right\}$. Hence (58) holds.

Assume that $\mathrm{Z}: \mathcal{P}_{o}^{n} \rightarrow\left\langle\mathcal{K}^{n},+\right\rangle$ is an $\operatorname{SL}(n)$ covariant Minkowski valuation. Now, we are going to derive some functional equations for support functions of $\mathrm{Z}$. These equations are closely related to those treated in Section 5 and will be often used. Let $s>0, \lambda \in(0,1)$, 
and denote by $H_{\lambda}$ the hyperplane passing through $o$ with normal vector $\lambda e_{1}-(1-\lambda) e_{2}$. Then

$$
\left(s T^{n}\right) \cap H_{\lambda}^{+}=s \phi_{\lambda} T^{n}, \quad\left(s T^{n}\right) \cap H_{\lambda}^{-}=s \psi_{\lambda} T^{n} \quad \text { and } \quad\left(s T^{n}\right) \cap H_{\lambda}=s \phi_{\lambda} T^{\prime},
$$

where $T^{\prime}=T^{n} \cap e_{1}^{\perp}$. So the valuation property of $\mathrm{Z}$ implies

$$
\mathrm{Z}\left(s T^{n}\right)+\mathrm{Z}\left(s \phi_{\lambda} T^{\prime}\right)=\mathrm{Z}\left(s \phi_{\lambda} T^{n}\right)+\mathrm{Z}\left(s \psi_{\lambda} T^{n}\right)
$$

The $\operatorname{SL}(n)$ covariance of $\mathrm{Z}$ therefore gives

$$
\begin{aligned}
& \mathrm{Z}\left(s T^{n}\right)+\lambda^{-1 / n} \phi_{\lambda} \mathrm{Z}\left(s \lambda^{1 / n} T^{\prime}\right) \\
& \quad=\lambda^{-1 / n} \phi_{\lambda} \mathrm{Z}\left(s \lambda^{1 / n} T^{n}\right)+(1-\lambda)^{-1 / n} \psi_{\lambda} \mathrm{Z}\left(s(1-\lambda)^{1 / n} T^{n}\right) .
\end{aligned}
$$

So from (4) and (5) we obtain

$$
\begin{aligned}
h_{\mathrm{Z}\left(s T^{n}\right)}(x)+\lambda^{-1 / n} h_{\mathrm{Z}\left(s \lambda^{1 / n} T^{\prime}\right)}\left(\phi_{\lambda}^{t} x\right) & \\
& =\lambda^{-1 / n} h_{\mathrm{Z}\left(s \lambda^{1 / n} T^{n}\right)}\left(\phi_{\lambda}^{t} x\right)+(1-\lambda)^{-1 / n} h_{\mathrm{Z}\left(s(1-\lambda)^{1 / n} T^{n}\right)}\left(\psi_{\lambda}^{t} x\right) .
\end{aligned}
$$

The SL $(n)$ covariant Minkowski valuation $\mathrm{Z}: \mathcal{P}_{o}^{n} \rightarrow\left\langle\mathcal{K}^{n},+\right\rangle$ gives rise to the following valuation $\hat{Z}$. For $n \geq 3$ and $1<k<n$ we can define, by Lemma 10, a map $\hat{Z}: \mathcal{P}_{o}^{k} \rightarrow$ $\left\langle\mathcal{K}^{k},+\right\rangle$ by

$$
\hat{\mathrm{Z} P}=\pi_{k}\left(\mathrm{Z}\left(\pi_{k}^{-1} P\right)\right),
$$

where $\pi_{k}: \operatorname{lin}\left\{e_{1}, \ldots, e_{k}\right\} \rightarrow \mathbb{R}^{k}$ denotes the projection of a vector to its first $k$ coordinates with respect to the standard basis. Note that $\pi_{k}$ is a linear bijection. It is easily seen that $\hat{Z}$ is in fact an $\operatorname{SL}(k)$ covariant Minkowski valuation. Furthermore, for $P \subset \operatorname{lin}\left\{e_{1}, \ldots, e_{k}\right\}$ we infer from (6) and Lemma 10 that

$$
h_{\mathrm{Z} P}(x)=h_{\hat{\mathrm{Z}}\left(\pi_{k} P\right)}\left(\pi_{k}\left(x_{1} e_{1}+\cdots+x_{k} e_{k}\right)\right), \quad x \in \mathbb{R}^{n} .
$$

Moreover, Corollary 2 shows that for $P \in \mathcal{P}_{o}^{k}$,

$$
\hat{\mathrm{Z}}\left(\phi_{\lambda} P\right)=\phi_{\lambda} \hat{\mathrm{Z}} P \quad \text { and } \quad \hat{\mathrm{Z}}\left(\psi_{\lambda} P\right)=\psi_{\lambda} \hat{\mathrm{Z}} P \text {, }
$$

as well as

$$
\hat{\mathrm{Z}}(s P)=s \hat{\mathrm{Z}} P
$$

for any $s>0$. In particular, from (4), (5), (59), and (63) we get

$$
h_{\hat{\mathrm{Z}}\left(s T^{k}\right)}(x)+h_{\hat{\mathrm{Z}}\left(s T^{\prime}\right)}\left(\phi_{\lambda}^{t} x\right)=h_{\hat{\mathrm{Z}}\left(s T^{k}\right)}\left(\phi_{\lambda}^{t} x\right)+h_{\hat{\mathrm{Z}}\left(s T^{k}\right)}\left(\psi_{\lambda}^{t} x\right) .
$$




\subsection{The crucial classification}

In this subsection we establish a theorem which has all the main results on covariant valuations stated in Sections 1 and 3 as consequences. We start by clarifying the behavior of SL $(n)$ covariant Minkowski valuations on lower dimensional sets.

Lemma 11. Suppose that $n \geq 3$ and $\mathrm{Z}: \mathcal{P}_{o}^{n} \rightarrow\left\langle\mathcal{K}^{n},+\right\rangle$ is an $\mathrm{SL}(n)$ covariant Minkowski valuation which is continuous at the line segment $\left[o, e_{1}\right]$. Then there exist non-negative constants $c_{1}$ and $c_{2}$ such that for every convex polytope $P \in \mathcal{P}_{o}^{n}$ of dimension less than $n$ one has

$$
\mathrm{Z} P=c_{1} P+c_{2}(-P)
$$

Proof. From Lemma 10 we know that there exist constants $c_{1}$ and $c_{2}$ such that $-c_{2} \leq c_{1}$ and $\mathrm{Z}\left[o, e_{1}\right]=\left[-c_{2} e_{1}, c_{1} e_{1}\right]$. By (4) it suffices to prove that $c_{1}, c_{2} \geq 0$ and

$$
h_{\mathrm{Z} P}-c_{1} h_{P}-c_{2} h_{-P}=0 \quad \text { for every } P \in \mathcal{P}_{o}^{n} \text { with } \operatorname{dim} P<n .
$$

We prove (66) by induction on dimension. If $\operatorname{dim} P=0$, then $\mathrm{Z} P=\{o\}$ by Lemma 10 and hence (66) obviously holds. It easily follows from (7) that

$$
h_{\left[-c_{2} e_{1}, c_{1} e_{1}\right]}=c_{1} h_{\left[o, e_{1}\right]}+c_{2} h_{-\left[o, e_{1}\right]} .
$$

Hence, by the definition of $c_{1}$ and $c_{2}$, equation (66) also holds for the segment $\left[o, e_{1}\right]$. Next, let $P$ be a line segment of the form $[o, x]$ for some non-zero $x \in \mathbb{R}^{n}$. Let $\vartheta \in \operatorname{SL}(n)$ be a rotation with $x=\|x\| \vartheta e_{1}$. Then the covariance of Z, (57), (5), and the already established equality (66) for $\left[o, e_{1}\right]$ give

$h_{\mathrm{Z}[o, x]}=\|x\| h_{\mathrm{Z}\left[o, e_{1}\right]} \circ \vartheta^{t}=\|x\|\left(c_{1} h_{\left[o, e_{1}\right]}+c_{2} h_{-\left[o, e_{1}\right]}\right) \circ \vartheta^{t}=c_{1} h_{[o, x]}+c_{2} h_{-[o, x]}$.

More generally, let $P \in \mathcal{P}_{o}^{n}$ be of the form $P=[-a x, b x]$ for some non-zero $x \in \mathbb{R}^{n}$ and positive constants $a$ and $b$. The valuation property, the fact that $Z\{o\}=\{o\}$ (which follows from Lemma 10), and (67) give

$$
h_{\mathrm{Z} P}=h_{\mathrm{Z}[o,-a x]}+h_{\mathrm{Z}[o, b x]}=c_{1} h_{P}+c_{2} h_{-P} .
$$

Thus (66) holds for 1-dimensional bodies.

Let $2 \leq k \leq n-1$ and assume that (66) holds for $(k-1)$-dimensional bodies. As before, define $\hat{\mathrm{Z}}: \mathcal{P}_{o}^{k} \rightarrow\left\langle\mathcal{K}^{k},+\right\rangle$ by $\hat{\mathrm{Z}} P=\pi_{k}\left(\mathrm{Z}\left(\pi_{k}^{-1} P\right)\right)$. Recall that $\hat{\mathrm{Z}}$ is an $\operatorname{SL}(k)$ covariant Minkowski valuation. Note that $\hat{Z}$ is also continuous at the line segment $\left[o, e_{1}\right]$. Moreover, since we assume that (66) holds for $(k-1)$-dimensional polytopes, we have

$$
h_{\hat{\mathrm{Z}} P}-c_{1} h_{P}-c_{2} h_{-P}=0 \quad \text { for every } P \in \mathcal{P}_{o}^{k} \text { with } \operatorname{dim} P<k .
$$

Define a function $f: \mathbb{R}_{+} \times \mathbb{R}^{k} \rightarrow \mathbb{R}$ by

$$
f(s, x)=h_{\hat{\mathbf{Z}}\left(s T^{k}\right)}(x)-c_{1} h_{s T^{k}}(x)-c_{2} h_{-s T^{k}}(x) .
$$

Let $\phi$ be a linear map with

$$
\phi e_{1}=e_{2}, \quad \phi e_{2}=e_{1}, \quad \phi e_{j}=e_{j} \quad \text { if } k \geq 3 \text { and } 3 \leq j \leq k .
$$


Clearly we have $\phi^{t}=\phi$ and $\phi T^{k}=T^{k}$. Recall that $n \geq 3$ and $k<n$. The $\operatorname{SL}(n)$ covariance of $\mathrm{Z}$ therefore implies $\hat{\mathrm{Z}}\left(\phi T^{k}\right)=\phi \hat{\mathrm{Z}} T^{k}$ and we deduce from (5) that

$$
f\left(1, e_{1}\right)=f\left(1, e_{2}\right) \text { and } f\left(1,-e_{1}\right)=f\left(1,-e_{2}\right) .
$$

From (64) and (4) one immediately deduces

$$
f(s, x)=s f(1, x) \quad \text { for every }(s, x) \in \mathbb{R}_{+} \times \mathbb{R}^{k} .
$$

Note that equation (65) holds in dimension $k$ for $\hat{Z}, c_{1}$ times the identity as well as $c_{2}$ times the reflection at the origin. Subtracting the respective equalities and using (68) therefore gives, for all $(s, x) \in \mathbb{R}_{+} \times \mathbb{R}^{k}$,

$$
f(s, x)=f\left(s, \phi_{\lambda}^{t} x\right)+f\left(s, \psi_{\lambda}^{t} x\right) .
$$

Thus $f$ satisfies the functional equation (25) for $p=q=0$. By appealing to (70) we can apply Lemma 7 for $r=1$ and conclude that for $x_{1}>x_{2}>0$,

$$
\begin{aligned}
f\left(s, x_{1} e_{1}+x_{2} e_{2}\right) & =s\left(x_{1}-x_{2}\right) f\left(1, e_{1}\right), \\
f\left(s,-x_{1} e_{1}-x_{2} e_{2}\right) & =s\left(x_{1}-x_{2}\right) f\left(1,-e_{1}\right) ;
\end{aligned}
$$

for $x_{2}>x_{1}>0$,

$$
\begin{aligned}
f\left(s, x_{1} e_{1}+x_{2} e_{2}\right) & =s\left(x_{2}-x_{1}\right) f\left(1, e_{2}\right), \\
f\left(s,-x_{1} e_{1}-x_{2} e_{2}\right) & =s\left(x_{2}-x_{1}\right) f\left(1,-e_{2}\right) ;
\end{aligned}
$$

and for $x_{1}, x_{2}>0$

$$
\begin{aligned}
f\left(s,-x_{1} e_{1}+x_{2} e_{2}\right) & =s\left[x_{2} f\left(1, e_{2}\right)+x_{1} f\left(1,-e_{1}\right)\right], \\
f\left(s, x_{1} e_{1}-x_{2} e_{2}\right) & =s\left[x_{1} f\left(1, e_{1}\right)+x_{2} f\left(1,-e_{2}\right)\right] .
\end{aligned}
$$

Define constants

$$
a=f\left(1, e_{1}\right) \quad \text { and } \quad b=f\left(1,-e_{1}\right) .
$$

Using these definitions, the symmetry relation (69), and the continuity of $f$, it is readily verified that for all $x_{1}, x_{2} \in \mathbb{R}$,

$$
\begin{aligned}
f\left(1, x_{1} e_{1}+x_{2} e_{2}\right)= & a\left[2 \max \left\{\left(x_{1}\right)_{+},\left(x_{2}\right)_{+}\right\}-\left(x_{1}\right)_{+}-\left(x_{2}\right)_{+}\right] \\
& +b\left[2 \max \left\{\left(x_{1}\right)_{-},\left(x_{2}\right)_{-}\right\}-\left(x_{1}\right)_{-}-\left(x_{2}\right)_{-}\right] .
\end{aligned}
$$

Relations (7), (8), and the definition of E (Sec. 2) imply, for $x \in \operatorname{lin}\left\{e_{1}, e_{2}\right\}$,

$$
f(1, x)=2 a h_{T^{2}}(x)+2 b h_{-T^{2}}(x)-a h_{\mathrm{E} T^{2}}(x)-b h_{\mathrm{E}\left(-T^{2}\right)}(x) .
$$

Assume first that $k=2$. By the definition of $f$, (70), and the homogeneity of E we have

$$
h_{\hat{\mathrm{Z}}\left(s T^{2}\right)}=\left(2 a+c_{1}\right) h_{s T^{2}}+\left(2 b+c_{2}\right) h_{-s T^{2}}-a h_{\mathrm{E}\left(s T^{2}\right)}-b h_{\mathrm{E}\left(-s T^{2}\right)} \text {. }
$$


From Lemma 3 we therefore know that for all $P \in \mathcal{P}_{o}^{2}$,

$$
h_{\hat{\mathrm{Z}} P}=\left(2 a+c_{1}\right) h_{P}+\left(2 b+c_{2}\right) h_{-P}-a h_{\mathrm{E} P}-b h_{\mathrm{E}(-P)} .
$$

For sufficiently small $\varepsilon>0$ define $P_{\varepsilon} \in \mathcal{P}_{o}^{2}$ as $P_{\varepsilon}=\left[o, e_{1}\right]-\varepsilon e_{1}+\varepsilon\left[-e_{2}, e_{2}\right]$. Note that $\lim _{\varepsilon \rightarrow 0} P_{\varepsilon}=\left[o, e_{1}\right]$. From (72) we infer by the continuity of $\hat{\mathrm{Z}}$ at $\left[o, e_{1}\right]$ and the fact that for every $\varepsilon$ the origin is an interior point of $P_{\varepsilon}$ that

$$
\begin{aligned}
c_{1} & =h_{\hat{\mathrm{Z}}\left[o, e_{1}\right]}\left(e_{1}\right)=\lim _{\varepsilon \rightarrow 0^{+}} h_{\hat{\mathrm{Z}} P_{\varepsilon}}\left(e_{1}\right)=\lim _{\varepsilon \rightarrow 0^{+}}\left(\left(2 a+c_{1}\right) h_{P_{\varepsilon}}\left(e_{1}\right)+\left(2 b+c_{2}\right) h_{-P_{\varepsilon}}\left(e_{1}\right)\right) \\
& =2 a+c_{1} .
\end{aligned}
$$

This shows that $a=0$. By performing the same computation but for $-e_{1}$ instead of $e_{1}$, one obtains $b=0$. Thus $h_{\hat{\mathrm{Z}} P}=c_{1} h_{P}+c_{2} h_{-P}$. From (62), we obtain $h_{\mathrm{Z} P}=c_{1} h_{P}+$ $c_{2} h_{-P}$ for all $P \subset \operatorname{lin}\left\{e_{1}, e_{2}\right\}$. Now Lemma 9 proves that (66) holds for all 2-dimensional polytopes.

Assume now that $k \geq 3$. Since $\pm e_{3}$ are fixpoints of both $\phi_{\lambda}$ and $\psi_{\lambda}$, equation (71) immediately shows that $f\left(s, \pm e_{3}\right)=0$ for all $s>0$. Define $\phi \in \operatorname{SL}(k)$ by

$$
\phi e_{1}=e_{2}, \quad \phi e_{2}=e_{3}, \quad \phi e_{3}=e_{1}, \quad \phi e_{l}=e_{l} \quad \text { for } 3<l \leq k \text { if } k>3 .
$$

Since $\phi^{t} T^{k}=T^{k}$ we deduce from (5) and the covariance of $\hat{Z}$ that

$$
\begin{aligned}
f\left(s, e_{1}\right) & =f\left(s, \phi e_{3}\right)=h_{\hat{\mathrm{Z}}\left(s T^{k}\right)}\left(\phi e_{3}\right)-c_{1} h_{s T^{k}}\left(\phi e_{3}\right)-c_{2} h_{-s T^{k}}\left(\phi e_{3}\right) \\
& =h_{\hat{\mathrm{Z}}\left(s T^{k}\right)}\left(e_{3}\right)-c_{1} h_{s T^{k}}\left(e_{3}\right)-c_{2} h_{-s T^{k}}\left(e_{3}\right)=f\left(s, e_{3}\right)=0 .
\end{aligned}
$$

If we replace in the last argument $e_{1}$ and $e_{3}$ by $-e_{1}$ and $-e_{3}$, respectively, then we see that $f\left(s,-e_{1}\right)=0$. From (69) we conclude that also $f\left(s, \pm e_{2}\right)=0$ for all $s>0$. Thus $f(s, x)=0$ for every $x \in \operatorname{lin}\left\{e_{1}, e_{2}\right\}$. Since $f(s, \pi x)=f(s, x)$ for every $\pi \in$ $\mathrm{SL}(k)$ which is induced by a permutation matrix, Lemma 5 implies that $f(s, x)=0$ for every $x \in \mathbb{R}^{k}$. Hence $h_{\hat{\mathrm{Z}}\left(s T^{k}\right)}=c_{1} h_{s T^{k}}+c_{2} h_{-s T^{k}}$ for all positive $s$. From Lemma 3 we therefore know that $h_{\hat{\mathrm{Z}} P}=c_{1} h_{P}+c_{2} h_{-P}$. As above, one deduces that (66) holds for all $k$-dimensional polytopes.

It remains to show that the constants $c_{1}$ and $c_{2}$ are non-negative. By (66), $c_{1} h_{P}+$ $c_{2} h_{-P}$ has to be a support function for each $P \in \mathcal{P}_{o}^{n}$ with dimension less than $n$. We infer from (3) that

$$
0 \leq c_{1}\left[h_{P}(x)+h_{P}(y)-h_{P}(x+y)\right]+c_{2}\left[h_{-P}(x)+h_{-P}(y)-h_{-P}(x+y)\right]
$$

for all $x, y \in \mathbb{R}^{n}$. Evaluate this inequality at the 2-dimensional standard simplex $T^{2}=$ $\operatorname{conv}\left\{o, e_{1}, e_{2}\right\}, x=e_{1}$, and $y=e_{2}$. Then (6) and (8) immediately imply that $c_{1} \geq 0$. Similarly, by looking at $P=-T^{2}, x=e_{1}$, and $y=e_{2}$, one sees that also $c_{2} \geq 0$.

We are now in a position to establish the main classification for $\operatorname{SL}(n)$ covariant Minkowski valuations. 
Theorem 8. Let $n \geq 3$. Suppose that $\mathrm{Z}: \mathcal{P}_{o}^{n} \rightarrow\left\langle\mathcal{K}^{n},+\right\rangle$ is an $\operatorname{SL}(n)$ covariant Minkowski valuation which is continuous at the line segment $\left[o, e_{1}\right]$ and such that the two functions

$$
s \mapsto h_{\mathrm{Z}\left(s T^{n}\right)}\left(e_{3}\right) \quad \text { and } \quad s \mapsto h_{\mathrm{Z}\left(s T^{n}\right)}\left(-e_{3}\right), \quad s>0,
$$

are bounded from below on some non-empty open intervals $I_{+} \subset \mathbb{R}_{+}$and $I_{-} \subset \mathbb{R}_{+}$, respectively. Then there exist non-negative constants $c_{1}$ and $c_{2}$ as well as constants $c_{3}, c_{4} \in$ $\mathbb{R}$ such that

$$
h_{\mathrm{Z} P}=c_{1} h_{P}+c_{2} h_{-P}+c_{3} h_{\Gamma_{+} P}+c_{4} h_{\Gamma_{+}(-P)} \quad \text { for every } P \in \mathcal{P}_{o}^{n} .
$$

Proof. Let $c_{1}$ and $c_{2}$ be the constants from Lemma 11 . For $(s, x) \in \mathbb{R}_{+} \times \mathbb{R}^{n}$ set

$$
f(s, x)=h_{\mathrm{Z}\left(s T^{n}\right)}(x)-c_{1} h_{s T^{n}}(x)-c_{2} h_{-s T^{n}}(x) .
$$

By assumption, the functions $f\left(\cdot, e_{3}\right)$ and $f\left(\cdot,-e_{3}\right)$ are bounded from below on some non-empty open intervals $I_{+} \subset \mathbb{R}_{+}$and $I_{-} \subset \mathbb{R}_{+}$, respectively. Note that for every $\pi \in \operatorname{SL}(n)$ which is induced by a permutation matrix we have

$$
f(s, \pi x)=f(s, x) .
$$

Equation (61) is true for $\mathrm{Z}, c_{1}$ times the identity as well as $c_{2}$ times the reflection at the origin. Subtracting the respective equalities and using Lemma 11 therefore gives

$$
f(s, x)=\lambda^{-1 / n} f\left(s \lambda^{1 / n}, \phi_{\lambda}^{t} x\right)+(1-\lambda)^{-1 / n} f\left(s(1-\lambda)^{1 / n}, \psi_{\lambda}^{t} x\right) .
$$

From Corollary 1 we deduce that $f\left(s, \pm e_{i}\right)=s^{n+1} f\left(1, \pm e_{i}\right)$. An application of Lemma 7 for $p=-1 / n, q=1 / n$, and $r=n+1$ yields for $x_{1}>x_{2}>0$,

$$
\begin{aligned}
f\left(s, x_{1} e_{1}+x_{2} e_{2}\right) & =\left(x_{1}+x_{2}\right) f\left(s, e_{1}\right), \\
f\left(s,-x_{1} e_{1}-x_{2} e_{2}\right) & =\left(x_{1}+x_{2}\right) f\left(s,-e_{1}\right) ;
\end{aligned}
$$

for $x_{2}>x_{1}>0$,

$$
\begin{aligned}
f\left(s, x_{1} e_{1}+x_{2} e_{2}\right) & =\left(x_{1}+x_{2}\right) f\left(s, e_{2}\right), \\
f\left(s,-x_{1} e_{1}-x_{2} e_{2}\right) & =\left(x_{1}+x_{2}\right) f\left(s,-e_{2}\right) ;
\end{aligned}
$$

and for $x_{1}, x_{2}>0$

$$
\begin{aligned}
f\left(s,-x_{1} e_{1}+x_{2} e_{2}\right) & =\left(x_{1}+x_{2}\right)^{-1}\left(x_{2}^{2} f\left(s, e_{2}\right)+x_{1}^{2} f\left(s,-e_{1}\right)\right), \\
f\left(s, x_{1} e_{1}-x_{2} e_{2}\right) & =\left(x_{1}+x_{2}\right)^{-1}\left(x_{1}^{2} f\left(s, e_{1}\right)+x_{2}^{2} f\left(s,-e_{2}\right)\right) .
\end{aligned}
$$

Being the sum of continuous functions, $f$ is continuous in $x$ for each fixed $s$. This continuity at $x e_{1}+x e_{2}$ immediately shows

$$
f\left(s, e_{1}\right)=f\left(s, e_{2}\right) \quad \text { and } \quad f\left(s,-e_{1}\right)=f\left(s,-e_{2}\right) .
$$

Define constants $c_{3}$ and $c_{4}$ by

$$
c_{3}=(n+1) ! f\left(1, e_{1}\right) \quad \text { and } \quad c_{4}=(n+1) ! f\left(1,-e_{1}\right) .
$$


An elementary calculation proves

$$
f\left(s, x_{1} e_{1}+x_{2} e_{2}\right)=c_{3} h_{\Gamma_{+}\left(s T^{n}\right)}\left(x_{1} e_{1}+x_{2} e_{2}\right)+c_{4} h_{\Gamma_{+}\left(-s T^{n}\right)}\left(x_{1} e_{1}+x_{2} e_{2}\right) .
$$

By what we already proved, the function $f$ satisfies the assumptions of Lemma 5 , as also does $c_{3} h_{\Gamma_{+}\left(s T^{n}\right)}(x)+c_{4} h_{\Gamma_{+}\left(-s T^{n}\right)}(x)$. Applying Lemma 5 to the difference of these two functions shows

$$
f(s, x)=c_{3} h_{\Gamma_{+}\left(s T^{n}\right)}(x)+c_{4} h_{\Gamma_{+}\left(-s T^{n}\right)}(x) \quad \text { for every } x \in \mathbb{R}^{n} .
$$

The definition of $f$ gives

$$
h_{\mathrm{Z}\left(s T^{n}\right)}=c_{1} h_{s T^{n}}+c_{2} h_{-s T^{n}}+c_{3} h_{\Gamma^{+}\left(s T^{n}\right)}+c_{4} h_{\Gamma^{+}\left(-s T^{n}\right)} \quad \text { for all } s>0 .
$$

From Lemma 3 we therefore know that

$$
h_{\mathrm{Z} P}=c_{1} h_{P}+c_{2} h_{-P}+c_{3} h_{\Gamma_{+} P}+c_{4} h_{\Gamma_{+}(-P)} .
$$

\subsection{Proofs of the main theorems}

Using the results of the previous subsection, we are now in a position to establish all theorems on SL( $n)$ covariant Minkowski valuations stated in Sections 1 and 3.

Proof of Theorem 6. Clearly, the identity, the reflection at the origin, and the asymmetric centroid body operator are continuous SL $(n)$ covariant Minkowski valuations. Assume that $\mathrm{Z}: \mathcal{K}_{o}^{n} \rightarrow \mathcal{K}^{n}$ is a continuous $\mathrm{SL}(n)$ covariant Minkowski valuation. The continuity of $\mathrm{Z}$ implies that the two functions

$$
s \mapsto h_{\mathrm{Z}\left(s T^{n}\right)}\left(e_{3}\right) \quad \text { and } \quad s \mapsto h_{\mathrm{Z}\left(s T^{n}\right)}\left(-e_{3}\right), \quad s>0,
$$

are continuous. Hence they are bounded from below on some non-empty open intervals $I_{+} \subset \mathbb{R}_{+}$and $I_{-} \subset \mathbb{R}_{+}$, respectively. Theorem 8 together with the continuity of $\mathrm{Z}$ and the fact that $\mathcal{P}_{o}^{n}$ is a dense subset of $\mathcal{K}_{o}^{n}$ show that there exist non-negative constants $c_{1}$ and $c_{2}$ as well as constants $\hat{c}_{3}, \hat{c}_{4} \in \mathbb{R}$ with

$$
h_{\mathrm{Z} K}=c_{1} h_{K}+c_{2} h_{-K}+\hat{c}_{3} h_{\Gamma_{+} K}+\hat{c}_{4} h_{\Gamma_{+}(-K)} \quad \text { for every } K \in \mathcal{K}_{o}^{n} .
$$

Set $c_{3}=\left(\hat{c}_{3}+\hat{c}_{4}\right) / 2$ and $c_{4}=\left(\hat{c}_{3}-\hat{c}_{4}\right) / 2$. Then it is easy to see that

$$
\hat{c}_{3} h_{\Gamma_{+} K}+\hat{c}_{4} h_{\Gamma_{+}(-K)}=c_{3} h_{\Gamma K}+c_{4} h_{\mathrm{m}(K)} .
$$

Consequently,

$$
h_{\mathrm{Z} K}=c_{1} h_{K}+c_{2} h_{-K}+c_{3} h_{\Gamma K}+c_{4} h_{\mathrm{m}(K)} \quad \text { for all } K \in \mathcal{K}_{o}^{n} .
$$

It remains to show that $c_{3}$ is non-negative. Suppose that $K$ is origin-symmetric. Then $\mathrm{m}(K)=o$ and the positive homogeneity of the symmetric centroid body operator implies that

$$
s^{-n-1} h_{\mathrm{Z}(s K)}=s^{-n}\left[c_{1} h_{K}+c_{2} h_{-K}\right]+c_{3} h_{\Gamma K} \quad \text { for all } s>0 .
$$


Thus for every positive $s$, the function $s^{-n}\left[c_{1} h_{K}+c_{2} h_{-K}\right]+c_{3} h_{\Gamma K}$ is a support function and hence is sublinear. The pointwise limit of sublinear functions is sublinear. So if $s$ tends to infinity we deduce that $c_{3} h_{\Gamma K}$ is sublinear, i.e.

$$
0 \leq c_{3}\left[h_{\Gamma K}(x)+h_{\Gamma K}(y)-h_{\Gamma K}(x+y)\right] \quad \text { for all } x, y \in \mathbb{R}^{n} .
$$

In particular, for $K=[-1,1]^{n}, x=e_{1}$, and $y=-e_{1}$, it follows that $c_{3} \geq 0$.

Proof of Theorem 5. Let $s>0$. Since the origin is contained in $\mathrm{Z}\left(s T^{n}\right)$, we have $h_{\mathrm{Z}\left(s T^{n}\right)}$ $\geq 0$. Hence the functions $h_{\mathrm{Z}\left(s T^{n}\right)}\left( \pm e_{3}\right)$ are bounded from below on $\mathbb{R}_{+}$. By Theorem 8 there exist non-negative constants $c_{1}$ and $c_{2}$ as well as constants $c_{3}, c_{4} \in \mathbb{R}$ with

$$
h_{\mathrm{Z} P}=c_{1} h_{P}+c_{2} h_{-P}+c_{3} h_{\Gamma_{+} P}+c_{4} h_{\Gamma_{+}(-P)} \quad \text { for every } P \in \mathcal{P}_{o}^{n} .
$$

It remains to show that the constants $c_{3}$ and $c_{4}$ are non-negative. Let $s>0$. Since the origin is contained in $\mathrm{Z}\left(s T^{n}\right)$, evaluating the last equation at $s T^{n}$ and $e_{1}$ gives

$$
0 \leq h_{\mathrm{Z}\left(s T^{n}\right)}\left(e_{1}\right)=s c_{1} h_{T^{n}}\left(e_{1}\right)+s^{n+1} c_{3} h_{\Gamma_{+} T^{n}}\left(e_{1}\right) .
$$

Since $h_{\Gamma_{+} T^{n}}\left(e_{1}\right)>0$, taking the limit $s \rightarrow \infty$ in $0 \leq s^{-n} c_{1}+c_{3} h_{\Gamma_{+} T^{n}}\left(e_{1}\right)$ proves $c_{3} \geq 0$. Similarly, by looking at $-e_{1}$ instead of $e_{1}$, we get $c_{4} \geq 0$.

Proof of Theorem 2. Theorem 2 is an immediate consequence of Theorem 5 and the denseness of the set $P_{o}^{n}$ in $\mathcal{K}_{o}^{n}$.

Acknowledgments. The work of the author was supported by the Austrian Science Fund (FWF), within the project "Minkowski valuations and geometric inequalities", Project number P22388N13.

\section{References}

[1] Alesker, S.: Continuous rotation invariant valuations on convex sets. Ann. of Math. (2) 149, 977-1005 (1999) Zbl 0941.52002 MR 1709308

[2] Alesker, S.: Description of translation invariant valuations on convex sets with solution of P. McMullen's conjecture. Geom. Funct. Anal. 11, 244-272 (2001) Zbl 0995.52001 MR 1837364

[3] Alesker, S.: Hard Lefschetz theorem for valuations, complex integral geometry, and unitarily invariant valuations. J. Differential Geom. 63, 63-95 (2003) Zbl 1073.52004 MR 2015260

[4] Alesker, S.: The multiplicative structure on continuous polynomial valuations. Geom. Funct. Anal. 14, 1-26 (2004) Zbl 1072.52011 MR 2053598

[5] Alesker, S.: Theory of valuations on manifolds. I. Linear spaces. Israel J. Math. 156, 311-339 (2006) Zbl 1132.52017 MR 2282381

[6] Alesker, S.: Theory of valuations on manifolds. II. Adv. Math. 207, 420-454 (2006) Zbl 1117.52016 MR 2264077

[7] Alesker, S.: Theory of valuations on manifolds. IV. New properties of the multiplicative structure. In: Geometric Aspects of Functional Analysis, Lecture Notes in Math. 1910, Springer, Berlin, 1-44 (2007) Zbl 1127.52016 MR 2347038

[8] Alesker, S., Bernstein, J.: Range characterization of the cosine transform on higher Grassmannians. Adv. Math. 184, 367-379 (2004) Zbl 1059.22013 MR 2054020 
[9] Alesker, S., Fu, J. H. G.: Theory of valuations on manifolds. III. Multiplicative structure in the general case. Trans. Amer. Math. Soc. 360, 1951-1981 (2008) Zbl 1130.52008 MR 2366970

[10] Bernig, A.: Valuations with Crofton formula and Finsler geometry. Adv. Math. 210, 733-753 (2007) Zbl 1128.53047 MR 2303237

[11] Bernig, A.: A Hadwiger-type theorem for the special unitary group. Geom. Funct. Anal. 19, 356-372 (2009) Zbl 1180.53076 MR 2545241

[12] Bernig, A.: A product formula for valuations on manifolds with applications to the integral geometry of the quaternionic line. Comment. Math. Helv. 84, 1-19 (2009) Zbl 1162.53055 MR 2466073

[13] Bernig, A., Bröcker, L.: Valuations on manifolds and Rumin cohomology. J. Differential Geom. 75, 433-457 (2007) Zbl 1117.58005 MR 2301452

[14] Bernig, A., Fu, J. H. G.: Convolution of convex valuations. Geom. Dedicata 123, 153-169 (2006) Zbl 1117.53054 MR 2299731

[15] Bernig, A., Fu, J. H. G.: Hermitian integral geometry. Ann. of Math. (2) 173, 907-945 (2011) Zbl 1230.52014 MR 2776365

[16] Campi, S., Gronchi, P.: The $L^{p}$-Busemann-Petty centroid inequality. Adv. Math. 167, 128 141 (2002) Zbl 1002.52005 MR 1901248

[17] Cianchi, A., Lutwak, E., Yang, D., Zhang, G.: Affine Moser-Trudinger and Morrey-Sobolev inequalities. Calc. Var. Partial Differential Equations 36, 419-436 (2009) Zbl 1202.26029 MR 2551138

[18] Dafnis, N., Paouris, G.: Small ball probability estimates, $\psi_{2}$-behavior and the hyperplane conjecture. J. Funct. Anal. 258, 1933-1964 (2010) Zbl 1189.52004 MR 2578460

[19] Fleury, B., Guedon, O., Paouris, G.: A stability result for mean width of $L_{p}$-centroid bodies. Adv. Math. 214, 865-877 (2007) Zbl 1132.52012 MR 2349721

[20] Fu, J. H. G.: Structure of the unitary valuation algebra. J. Differential Geom. 72, 509-533 (2006) Zbl 1096.52003 MR 2219942

[21] Gardner, R. J.: Geometric Tomography. 2nd ed., Encyclopedia Math. Appl. 58, Cambridge Univ. Press, Cambridge (2006) Zbl 1102.52002 MR 2251886

[22] Gruber, P.: Convex and Discrete Geometry. Springer, Berlin (2007) Zbl 1139.52001 MR 2335496

[23] Haberl, C.: $L_{p}$ intersection bodies. Adv. Math. 217, 2599-2624 (2008) Zbl 1140.52003 MR 2397461

[24] Haberl, C.: Star body valued valuations. Indiana Univ. Math. J. 58, 2253-2276 (2009) Zbl 1183.52003 MR 2583498

[25] Haberl, C.: Blaschke valuations. Amer. J. Math. 133, 717-751 (2011) Zbl 1229.52003 MR 2808330

[26] Haberl, C., Ludwig, M.: A characterization of $L_{p}$ intersection bodies. Int. Math. Res. Notices 2006, art. ID 10548, 29 pp. Zbl 1115.52006 MR 2250020

[27] Haberl, C., Schuster, F. E.: Asymmetric affine $L_{p}$ Sobolev inequalities. J. Funct. Anal. 257, 641-658 (2009) Zbl 1180.46023 MR 2530600

[28] Haberl, C., Schuster, F. E.: General $L_{p}$ affine isoperimetric inequalities. J. Differential Geom. 83, 1-26 (2009) Zbl 1185.52005 MR 2545028

[29] Hug, D., Schneider, R., Schuster, R.: The space of isometry covariant tensor valuations. Algebra i Analiz 19, 194-224 (2007) Zbl 1134.52008 MR 2319515

[30] Kiderlen, M., Weil, W.: Measure-valued valuations and mixed curvature measures of convex bodies. Geom. Dedicata 76, 291-329 (1999) Zbl 0933.52014 MR 1702303

[31] Klain, D. A.: Star valuations and dual mixed volumes. Adv. Math. 121, 80-101 (1996) Zbl 0858.52003 MR 1399604 
[32] Klain, D. A.: Invariant valuations on star-shaped sets. Adv. Math. 125, 95-113 (1997) Zbl 0889.52007 MR 1427802

[33] Klain, D. A.: Even valuations on convex bodies. Trans. Amer. Math. Soc. 352, 71-93 (2000) Zbl 0940.52002 MR 1487620

[34] Klain, D. A., Rota, G.-C.: Introduction to Geometric Probability. Cambridge Univ. Press, Cambridge (1997) Zbl 0896.60004 MR 1608265

[35] Ludwig, M.: Projection bodies and valuations. Adv. Math. 172, 158-168 (2002) Zbl 1019.52003 MR 1942402

[36] Ludwig, M.: Valuations of polytopes containing the origin in their interiors. Adv. Math. 170, 239-256 (2002) Zbl 1015.52012 MR 1932331

[37] Ludwig, M.: Ellipsoids and matrix valued valuations. Duke Math. J. 119, 159-188 (2003) Zbl 1033.52012 MR 1991649

[38] Ludwig, M.: Minkowski valuations. Trans. Amer. Math. Soc. 357, 4191-4213 (2005) Zbl 1077.52005 MR 2159706

[39] Ludwig, M.: Intersection bodies and valuations. Amer. J. Math. 128, 1409-1428 (2006) Zbl 1115.52007 MR 2275906

[40] Ludwig, M., Reitzner, M.: A characterization of affine surface area. Adv. Math. 147, 138-172 (1999) Zbl 0947.52003 MR 1725817

[41] Ludwig, M., Reitzner, M.: A classification of SL $(n)$ invariant valuations. Ann. of Math. (2) 172, 1219-1267 (2010) Zbl 1223.52007 MR 2680490

[42] Lutwak, E., Yang, D., Zhang, G.: $L_{p}$ affine isoperimetric inequalities. J. Differential Geom. 56, 111-132 (2000) Zbl 1034.52009 MR 1863023

[43] Lutwak, E., Yang, D., Zhang, G.: A new ellipsoid associated with convex bodies. Duke Math. J. 104, 375-390 (2000) Zbl 0974.52008 MR 1781476

[44] Lutwak, E., Yang, D., Zhang, G.: The Cramer-Rao inequality for star bodies. Duke Math. J. 112, 59-81 (2002) Zbl 1021.52008 MR 1890647

[45] Lutwak, E., Yang, D., Zhang, G.: Sharp affine $L_{p}$ Sobolev inequalities. J. Differential Geom. 62, 17-38 (2002) Zbl 1073.46027 MR 1987375

[46] Lutwak, E., Yang, D., Zhang, G.: Moment-entropy inequalities. Ann. Probab. 32, 757-774 (2004) Zbl 1053.60004 MR 2039942

[47] Lutwak, E., Yang, D., Zhang, G.: Orlicz projection bodies. Adv. Math. 223, 220-242 (2010) Zbl pre05643962 MR 2563216

[48] Lutwak, E., Yang, D., Zhang, G.: Orlicz centroid bodies. J. Differential Geom. 84, 365-387 (2010) Zbl 1206.49050 MR 2652465

[49] Lutwak, E., Zhang, G.: Blaschke-Santaló inequalities. J. Differential Geom. 47, 1-16 (1997) Zbl 0906.52003 MR 1601426

[50] McMullen, P.: Valuations and dissections. In: Handbook of Convex Geometry, Vol. B, P. Gruber and J. Wills (eds.), North-Holland, Amsterdam, 933-990 (1993) Zbl 0791.52014 MR 1243000

[51] McMullen, P., Schneider, R.: Valuations on convex bodies. In: Convexity and its Applications, P. Gruber and J. Wills (eds.), Birkhäuser, 170-247 (1983) Zbl 0534.52001 MR 0731112

[52] Molchanov, I.: Convex and star-shaped sets associated with multivariate stable distributions. I. Moments and densities. J. Multivariate Anal. 100, 2195-2113 (2009) Zbl 1196.60029 MR 2560363

[53] Paouris, G.: Concentration of mass on convex bodies. Geom. Funct. Anal. 16, 1021-1049 (2006) Zbl 1114.52004 MR 2276533

[54] Ryabogin, D., Zvavitch, A.: The Fourier transform and Firey projections of convex bodies. Indiana Univ. Math. J. 53, 667-682 (2004) Zbl 1062.52004 MR 2086696 
[55] Schneider, R.: Convex Bodies: the Brunn-Minkowski Theory. Cambridge Univ. Press, Cambridge (1993) Zbl 0798.52001 MR 1216521

[56] Schneider, R., Schuster, F. E.: Rotation equivariant Minkowski valuations. Int. Math. Res. Notices 2006, art. ID 72894, 20 pp. Zbl 1127.52017 MR 2272092

[57] Schuster, F. E.: Crofton measures and Minkowski valuations. Duke Math. J. 154, 1-30 (2010) Zbl 1205.52004 MR 2668553

[58] Schuster, F. E., Wannerer, T.: GL(n) contravariant Minkwoski valuations. Trans. Amer. Math. Soc. 364, 815-826 (2012) Zbl pre06009180 MR 2846354

[59] Stancu, A.: The discrete planar $L_{0}$-Minkowski problem. Adv. Math. 167, 160-174 (2002) Zbl 1005.52002 MR 1901250

[60] Stancu, A.: On the number of solutions to the discrete two-dimensional $L_{0}$-Minkowski problem. Adv. Math. 180, 290-323 (2003) Zbl 1054.52001 MR 2019226

[61] Steineder, C.: Subword complexity and projection bodies. Adv. Math. 217, 2377-2400 (2008) Zbl 1203.52012 MR 2388098

[62] Thompson, A. C.: Minkowski Geometry, Encyclopedia Math. Appl. 63, Cambridge Univ. Press, Cambridge (1996) Zbl 0868.52001 MR 1406315

[63] Tsang, A.: Valuations on $L^{p}$-spaces. Int. Math. Res. Notices 2010, no. 20, 3993-4023 Zbl 1211.52013 MR 2738348

[64] Werner, E., Ye, D.: New $L_{p}$ affine isoperimetric inequalities. Adv. Math. 218, 762-780 (2008) Zbl 1155.52002 MR 2414321

[65] Yaskin, V., Yaskina, M.: Centroid bodies and comparison of volumes. Indiana Univ. Math. J. 55, 1175-1194 (2006) Zbl 1102.52005 MR 2244603

[66] Zhang, G.: The affine Sobolev inequality. J. Differential Geom. 53, 183-202 (1999) Zbl 1040.53089 MR 1776095 